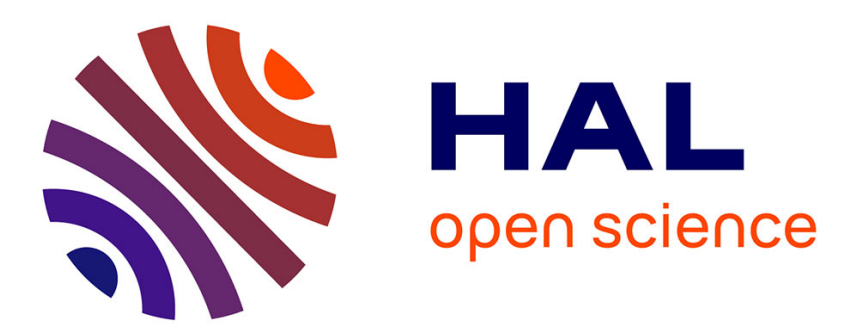

\title{
Asymptotic solution to the viscous/inertial flow in wavy channels with permeable walls
}

Mojdeh Rasoulzadeh, Mikhail Panfilov

\section{To cite this version:}

Mojdeh Rasoulzadeh, Mikhail Panfilov. Asymptotic solution to the viscous/inertial flow in wavy channels with permeable walls. Physics of Fluids, 2018, 30 (10), pp.106604. 10.1063/1.5041748 . hal-03265507

\section{HAL Id: hal-03265507 \\ https://hal.univ-lorraine.fr/hal-03265507}

Submitted on 22 Jun 2021

HAL is a multi-disciplinary open access archive for the deposit and dissemination of scientific research documents, whether they are published or not. The documents may come from teaching and research institutions in France or abroad, or from public or private research centers.
L'archive ouverte pluridisciplinaire HAL, est destinée au dépôt et à la diffusion de documents scientifiques de niveau recherche, publiés ou non, émanant des établissements d'enseignement et de recherche français ou étrangers, des laboratoires publics ou privés. 


\title{
Asymptotic solution to the viscous/inertial flow in wavy channels with permeable walls
}

\author{
Mojdeh Rasoulzadeh ${ }^{1}$, and Mikhail Panfilov ${ }^{2}$ \\ ${ }^{1}$ Department of Mathematics, The University of Alabama, Tuscaloosa, Alabama 35487, USA; \\ mrasoulzadeh@ua.edu \\ ${ }^{2}$ Institut Elie Cartan, CNRS/Université de Lorraine, Nancy, France
}

\begin{abstract}
Flow in a wavy channel immersed in the porous medium can be described neither as the flow in porous media nor as the flow in a regular pipe. The flow through the walls and the irregular wavy geometry of the walls result in non-negligible inertial and visco-inertial effects. The quadratic and cubic corrections to Darcys law proposed since Forchheimer have always been the subject of debate. In this paper, the complete set of Navier-Stokes equations is solved in a channel with wavy walls immersed in a porous medium. The asymptotic solution is obtained using the perturbation method considering the channels aspect ratio as the perturbation parameter. The two-scale homogenization technique is used to capture the effect of the wavy corrugations on the overall flow in the channel. The averaged pressure drop along the channel is represented as quadratic and cubic corrections to the linear law for a cylindrical and parallel-plate wavy channel. Published by AIP Publishing. https://doi.org/10.1063/1.5041748
\end{abstract}

\section{INTRODUCTION}

Investigating the flow of a viscous fluid in a channel with wavy permeable walls immersed in porous medium is of great significance due to its direct application to many scientific and industrial problems such as flow in blood vessels with nutrient exchange to the surrounding organs through the walls, filtration systems, fractured subsurface reservoirs, and many other applications. The classical Poiseuille flow in the channels cannot describe this problem due to the variable cross section and presence of fluid flow through the walls. This problem is also different from the problem of flow in heterogeneous porous media. The channel creates preferential flow paths in the porous medium and influences the overall flow regime. Darcys law does not hold in the channel. Several phenomena cause deviations from Darcys law. First, the flow in the channels open width happens at relatively high velocities, while in the porous rock seepage flow occurs with negligible inertial 
effects. The momentum conservation laws in the channel and in the porous rock are different one from another. The nonzero Reynolds number in the channel determines the non-neglecting nonlinear inertial effects. The second phenomenon is the external flux crossing the channels boundary, which influences both the average mass balance and momentum balance. Fluid exchange between the porous rock and the channel depends on the flow field in the porous rock, high inertial effects in the channel, the pressure drop along the channel, and the geometry of the channels boundaries. The third phenomenon is the wavy, irregular, and nonparallel-plate shape of the boundaries, which affects the overall flow regime and the averaged flow model. The characteristics of flow in the channel and, consequently, in the porous host rock are highly influenced by the presence of these phenomena.

Numerical simulation of this problem requires integrating several complex methodological tools, including a sophisticated gridding tool to represent the channels geometry and a robust solver to take care of different physical flow phenomena both inside and outside the channel as well as the different time/spatial scales to deal with the complexities present in the problem. Analytic solutions will ideally exclude model construction difficulties and will improve our understanding of the dynamics of flow at the scale of the channel as well as at the scale of the porous host.

While a general comprehensive model of flow in channels with wavy permeable walls is still an ongoing area of research, several approaches have focused on one of the aspects of this problem independent from the other aspects. In the following, some of these approaches are reviewed:

Parallel-plate impermeable walls. One of the oversimplifications is to neglect the influx through the walls and to assume a parallel-plate geometry for the walls. For dominant viscous forces over inertial forces, the Stokes flow in a regular channel or pipe with impermeable walls averaged over the channels open width represents the Poiseuille law, which yields to the linear proportionality of the averaged velocity and pressure gradient along the channel [Savage (1964), Landau (1974), Pfitzner (1976), Katsuhiko (1976), Bennett and Myers (1982), Sutera and Skalak (1993), and Zimmerman and Bodvarsson (1996a)]. With dominant inertial forces, quadratic and cubic corrections to this linear law were proposed by Forchheimer (1901). Since Forchheimer, there has been a large debate on the use of quadratic or cubic corrections to the linear law. Some researchers, such as Mei and Auriault (1991) and Skjetne and Auriault (1999), showed that only cubic correction is required, where they applied homogenization theory to Navier-Stokes equations in a periodic cell. Some researchers like Whitaker (1996) and Chen et al. (2001) used the volume averaging method and homogenization theory and showed that only quadratic correction is required.

Parallel-plate permeable walls. In a channel with permeable walls, the permanent inflow from the ambient porous rocks produces a progressive increase of the flow velocity and the Reynolds number along the channels length. Therefore, Reynolds can reach sufficiently high values at which the inertial effects should be conserved in the model. Berman (1953) was the first to study the system of the Navier-Stokes equations for a rectangular long channel with porous walls. He proposed a similarity solution, in terms of stream functions under which the Navier-Stokes equations reduce to a nonlinear fourth-order ordinary differential equation with two boundary conditions at each wall. This equation, called the Bermans problem, is perturbed by the influx Reynolds as the 
small perturbation parameter. Several authors studied Bermans solution, in particular, Sellars (1955), Yuan (1956), White et al. (1958), Proudman (1960), Wah (1964), Robinson (1976), and Zaturska et al. (1988). If the influx Reynolds is small enough, there is only one solution. Increasing walls Reynolds, several bifurcations will appear giving rise to new branches of solutions. In this case, the small perturbation parameter is the inverse of the Reynolds number. Several authors investigated the flow in a pipe with permeable walls for the applications to the horizontal wells [Berman (1958), Ihara et al. (1994), Ouyang (1998), and Ouyang et al. (1998)]. Experimental investigations of flow within parallel-plate permeable walls propose the mechanistic models connected to the experimental data via the empirical coefficients. Yuan (1955) investigated the importance and the effect of the uniform injection and suction through thewalls on the overall flow experimentally. Ouyang (1998) and Ouyang et al. (1998) presented the momentum balance equation, which contains a modified friction factor for the perforated pipe. They showed that the wall inflow or outflow depends on the flow regime: it increases the walls friction for laminar flow and decreases the walls friction for turbulent flow.

Wavy impermeable walls. Several researchers have investigated experimentally, the influence of roughness or corrugation of the channels geometry on the averaged flow model. In Chen (1966) and Crosnier et al. (1970), experimental data were obtained for the pressure drop of a divergent confined radial flow between two parallel disc-shaped fractures with rough surfaces. It was shown that the quadratic deviation from the laminar flow regime appears. The empirical corrections to the mean fracture aperture were proposed by several researchers [Katsuhiko (1976), Keller (1998), Oron and Berkowitz (1998), Renshaw (1995), and Konzuk and Kueper (2004)]. The effect of inertia upon the flow in a thin rough fracture was numerically investigated by Skjetne et al. (1999), Zimmerman and Yeo (2000), Sisavath et al. (2003), Panfilov and Fourar (2006), and Mourzenko et al. (2018). Several analytical solutions are obtained for the flow in wavy channels [Adler and Thovert (1999), Wierschem et al. (2003), Sisavath et al. (2003), Malevich et al. (2006), Heining et al. (2012), and Adler et al. (2013)], where the solution to Stokes or Navier-Stokes equations was obtained in terms of the rough wall geometry oscillations. The averaged pressure gradient was proposed as the quadratic and/or cubic corrections to Darcys law. Several analytical models addressed channels with sinusoidal walls [Burns and Parkes (1967), Manton (1971), Lahbabi and Chang (1986), Zimmerman and Bodvarsson (1996b), Deiber and Schowalter (1979), Nishimura et al. (1984), Ge (1997), Sisavath et al. (2001), Sureshkumar (2001), Brush and Thomson (2003), Haroun (2007), and Shenoy et al. (2016)].

This paper is a sequel to Bues et al. (2004), which was devoted to the problem of flow in a wavy disc with impermeable walls. We apply the same approach to investigate the flow in a wavy channel with a slip boundary condition on the permeable walls. In this paper, the channel is assumed to be cylindrical or parallel-plate with wavy walls. The complete set of Navier-Stokes equations are solved analytically in the channel using the two-scale asymptotic method: the solution is a double asymptotic series expansion with respect to the small channels aperture and the small period of wall corrugation. The model is averaged over the channels open width. The averaged model contains the corrections to Darcys law, which result from the effect of the fluid flowthrough 
thewalls and the irregular geometry of the channel. Using the average model, the characteristics of flow in wavy channels are investigated. We show that the geometry of the channel and permeable or impermeable walls result in different averaged flow regimes.

\section{CYLINDRICAL WAVY CHANNELS}

We consider the problem of flow in an axis-symmetric cylindrical channel with permeablewavywalls, where the longitudinal axis of the channel is along the $X$ axis as shown in Figs. 1 and 2 . The channel is immersed in a porous medium containing a fluid. The walls are permeable and authorize the fluid to pass through. As shown in Fig. 2, the channels wavy geometry is represented by the domain $\left\{0<R<R_{w} \phi(X), 0<\theta<2 \pi, 0<X<L\right\}$, where $\phi(X)$ is a periodic function with period $l$ and mean value 1 . We assume that at the inlet $\phi(0)=1$. In case when $\phi(X)=1$, the channel will be a regular infinite cylinder. $R_{w}$ is the mean radius of the cylindrical channel and the characteristic length of the channel is $L$. We consider the complete set of axis-symmetric Navier-Stokes equations for steady-state flow of an incompressible fluid with density $\rho$ and dynamic viscosity $\mu$ in the cylindrical coordinate system. We assume that the influx to the inlet of the channel at $X=0$ is $Q^{0}$.

The boundary condition on the channels inlet imposes the given mean velocity $U^{0}$ (or flow rate $Q^{0}$ ) as following:

$$
U^{\circ} \equiv \frac{2}{\left(R_{w} \phi(0)\right)^{2}} \int_{0}^{R_{w} \phi(0)} U_{X}(R, 0) R d R .
$$

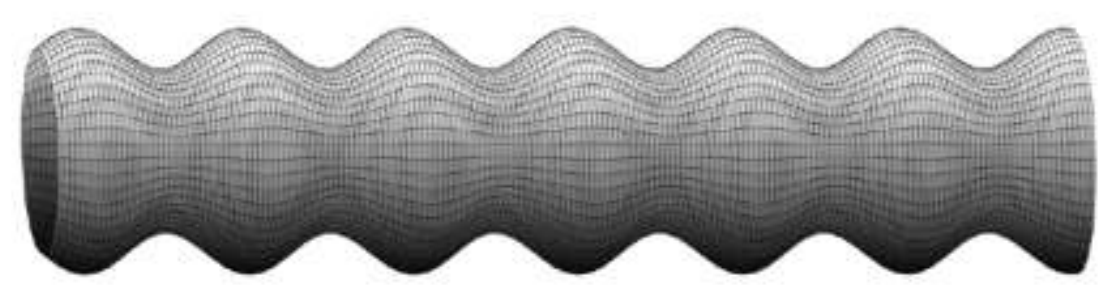

FIG. 1. The schematic of a cylindrical channel with wavy walls.

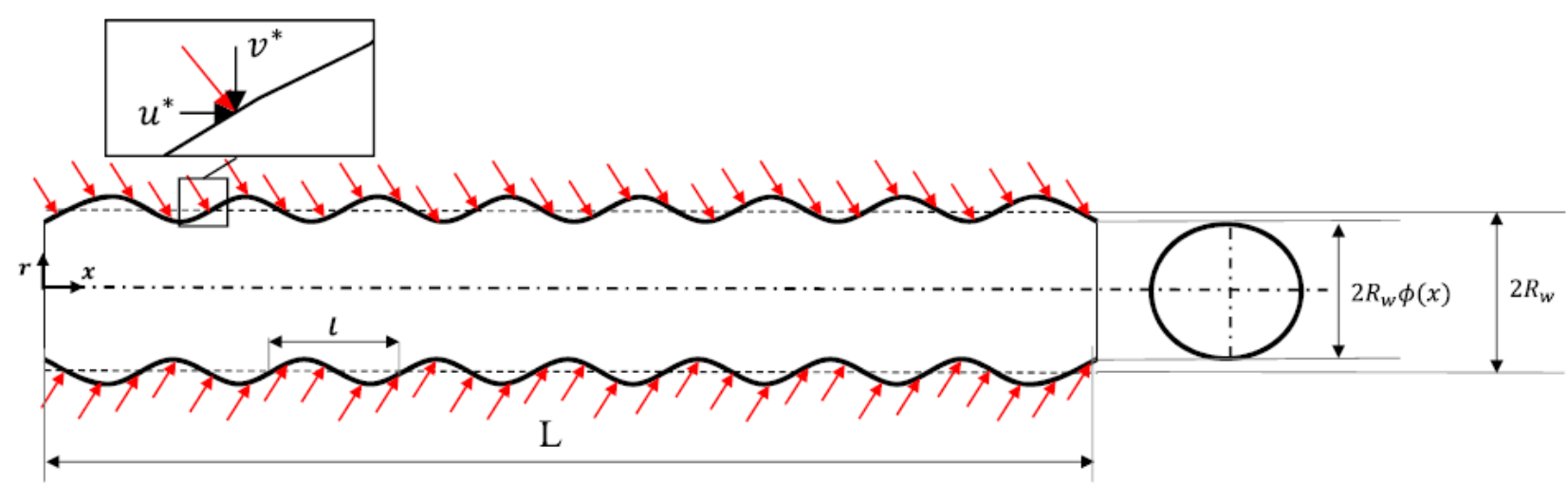

FIG. 2. Cylindrical wavy channel with uniform fluid flow through the walls. 
The channels boundary is penetrable to fluid to and from the host porous rock. We assume a uniform fluid exchange between the porous host and the channel along the channel walls with constant radial and axial component of $\left(U_{R}^{*}, U_{X}^{*}\right)$. Let us introduce the following dimensionless variables:

$$
x=\frac{X}{L}, \quad r=\frac{R}{R_{u}}, \quad p=\frac{P}{p^{\circ}}, \quad u=\frac{U_{X}}{U^{\circ}}, \quad v=\frac{U_{R}}{U^{\circ}},
$$

where $P, U_{X}$, and $U_{R}$ are pressure, axial, and radial fluid velocity, respectively. $p, u$, and $v$ are the dimensionless pressure, dimensionless axial, and dimensionless radial velocity. The characteristic pressure $p^{0}$ is determined as the pressure drop along the length $L$ caused by a Poiseuille flow at velocity $U^{0}: p^{0}=\frac{\mu U^{0} L}{R_{w}^{2}}$.

. Along with the dimensionless variables, we introduce three dimensionless parameters as

$$
\omega=\frac{R_{w}}{L}, \quad \varepsilon=\frac{\ell}{L}, \quad \operatorname{Re}=\frac{\rho U^{\circ} R_{w}}{\mu} .
$$

We assume that the parameters $\omega$ and $\varepsilon$ are small so that:

$$
0<\omega, \varepsilon<<1
$$

The ratio between them determines various regimes of flow. In particular, two limit cases reduce to a channel with straight walls:

- if $\omega / \varepsilon \gg>1$, then the channel's aperture is enormous, and the corrugations can be neglected, Fig.3.(a).

- if $\omega / \varepsilon<<1$, then the corrugation period is very large, Fig.3.(c).

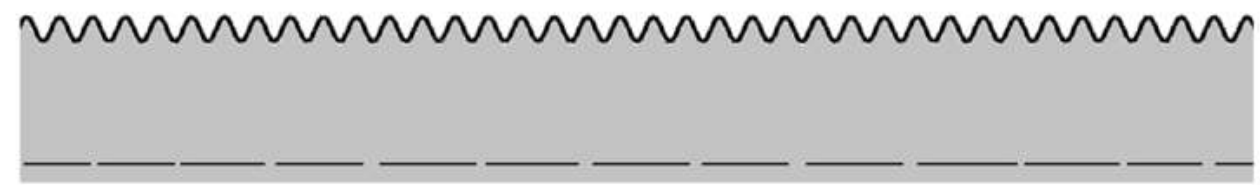

(a)

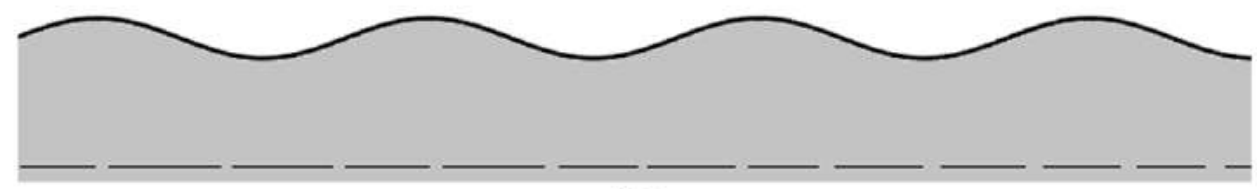

(b)

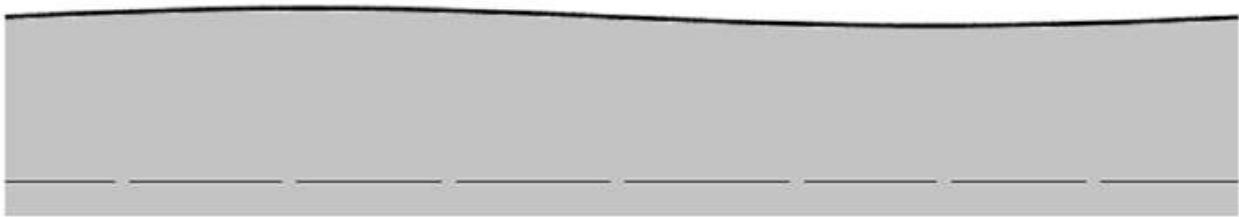

(c)

FIG. 3. Limit cases of the ratio between $\omega$ and $\varepsilon$ : (a) $\omega / \varepsilon \gg 1$, (b) $\omega \sim \varepsilon^{\alpha}$, $\alpha \sim 1$, and (c) $\omega / \varepsilon \ll 1$. 
Contrarily, the Reynolds number Re may take various values, from zero to several hundred, while the flow remains stable. In the dimensionless domain $\mathrm{W}(r, x)=\{0<r<\phi(x), 0<x<1\}$ with $\phi(x)$ being a periodic function of period $\varepsilon$ and the mean value 1, the steady-state mass conservation and Navier Stokes equations will reduce to

$$
\begin{gathered}
\omega \frac{\partial u}{\partial x}+\frac{1}{r} \frac{\partial(r v)}{\partial r}=0, \\
\operatorname{Re}\left(\omega u \frac{\partial u}{\partial x}+v \frac{\partial u}{\partial r}\right)=-\frac{\partial p}{\partial x}+\left[\omega^{2} \frac{\partial^{2} u}{\partial x^{2}}+\frac{1}{r} \frac{\partial}{\partial r}\left(r \frac{\partial u}{\partial r}\right)\right] . \quad(4 \mathrm{~b}) \\
\operatorname{Re}\left(\omega u \frac{\partial v}{\partial x}+v \frac{\partial v}{\partial r}\right)=-\frac{1}{\omega} \frac{\partial p}{\partial r}+\left[\omega^{2} \frac{\partial^{2} v}{\partial x^{2}}+\frac{1}{r} \frac{\partial}{\partial r}\left(r \frac{\partial v}{\partial r}\right)-\frac{v}{r^{2}}\right] .
\end{gathered}
$$

The dimensionless boundary conditions will rewrite to

$$
\begin{gathered}
1=2 \int_{0}^{\phi(0)} r u(r, 0) d r=2 \int_{0}^{1} r u(r, 0) d r, \\
\left.\frac{\partial u}{\partial r}\right|_{r=0}=0,\left.\quad v\right|_{r=0}=0, \\
\left.u\right|_{r=\phi(x)}=\omega u^{*},\left.\quad v\right|_{r=\phi(x)}=-\omega v^{*},
\end{gathered}
$$

where $\omega u^{*}=U_{X}^{*} / U^{\circ}, \omega v^{*}=U_{R}^{*} / U^{\circ}$ are two components of the inflow velocity through the fracture boundary. The condition (3.5a) results from (3.1), conditions (3.5b) means the symmetry with respect to the fracture axis. The presence of the small parameter $\omega$ in the relations for the inflow velocity means that it is assumed to be small.

This system possesses an integral relation which represents the mass balance between the external flux and the internal flow

$$
\int_{0}^{\phi(x)} r u(r, x) d r=F_{0}(x)+\omega F_{1}(x) .
$$

with

$$
F_{0}(x) \equiv \frac{1}{2}+v^{*} \int_{0}^{x} \phi(\bar{x}) d \bar{x}, \quad F_{1}(x) \equiv \frac{u^{*}}{2}\left(\phi^{2}(x)-\phi^{2}(0)\right) .
$$

Equation ( 6 ) is the average mass conservation, which says that the mass is not conserved due to the cross-flow through the walls. In other words, the flow as a function of $x$ at each point on the length of well results from the sum of inlet flow and inflow through the walls.

The system of boundary conditions (5a)-(5c) and (6) is not sufficient to determine the unique solution.

In this study, the objective is to analyze the behavior of a family of solutions. For simulations, the uniqueness may be reached by replacing condition (5a) imposed on the average velocity by the condition on the velocity at the inlet section.

The analysis is performed by applying the asymptotic method: the solution is searched in the form of the asymptotic expansion with respect to small parameters $\omega$ and $\varepsilon$. In the limit $\varepsilon \rightarrow 0$, we will obtain a system that is homogenized along $x$. The limit $\omega \rightarrow 0$ formally means the elimination of the transverse variable $r$, which means the homogenization along $r$. Thus the zero term of the asymptotic expansion is expected to be equal to the averaged 
value. Hence, the technique of the suggested method is close to those procedures which are applied in the homogenization theory. The flow equations averaged over $r$ represent the main interest of this paper. Owing to the particularity of the method used, the solutions obtained do not need to provide an exact detailed flow pattern, but they are expected to exhibit adequate homogenized behavior.

\section{A. Asymptotic solution}

Let us introduce the regular asymptotical expansion of $\mathcal{F}=\{u, v, p\}$ with respect to the small parameter $\omega$ as below:

$$
\mathcal{F}(r, x)=\mathcal{F}_{0}(r, x)+\omega \mathcal{F}_{1}(r, x)+\omega^{2} \mathcal{F}_{2}(r, x)+\cdots,
$$

The zeroth order approximation for $u_{0}, v_{0}, p_{0}$ gives

$$
\begin{gathered}
\frac{1}{r} \frac{\partial\left(r v_{0}\right)}{\partial r}=0, \quad \frac{\partial p_{0}}{\partial r}=0, \\
\operatorname{Re} v_{0} \frac{\partial u_{0}}{\partial r}=-\frac{\partial p_{0}}{\partial x}+\frac{1}{r} \frac{\partial}{\partial r}\left(r \frac{\partial u_{0}}{\partial r}\right), \\
\left.v_{0}\right|_{r=\phi}=0,\left.\quad u_{0}\right|_{r=\phi}=0,\left.\quad v_{0}\right|_{r=0}=0, \\
\left.\frac{\partial u_{0}}{\partial r}\right|_{r=0}=0, \quad \int_{0}^{\phi} r u_{0}(x, r) d r=F_{0}(x) .
\end{gathered}
$$

which directly gives $v_{0} \equiv 0$.

The first order approximation gives for $u_{1}, v_{1}, p_{1}$ as

$$
\begin{gathered}
\frac{1}{r} \frac{\partial\left(r v_{1}\right)}{\partial r}+\frac{\partial u_{0}}{\partial x}=0 \\
\operatorname{Re}\left(u_{0} \frac{\partial u_{0}}{\partial x}+v_{1} \frac{\partial u_{0}}{\partial r}\right)=-\frac{\partial p_{1}}{\partial x}+\frac{1}{r} \frac{\partial}{\partial r}\left(r \frac{\partial u_{1}}{\partial r}\right), \\
\frac{\partial p_{1}}{\partial r}=0,\left.\quad \frac{\partial u_{1}}{\partial r}\right|_{r=0}=0,\left.\quad v_{1}\right|_{r=0}=0, \\
\left.u_{1}\right|_{r=\phi(x)}=u^{*},\left.\quad v_{1}\right|_{r=\phi(x)}=-v^{*}, \quad \int_{0}^{\phi(x)} r u_{1}(x, r) d r=F_{1}(x) .
\end{gathered}
$$

The second order approximation gives for $u_{2}, v_{2}, p_{2}$ as

$$
\begin{gathered}
\frac{1}{r} \frac{\partial\left(r v_{2}\right)}{\partial r}+\frac{\partial u_{1}}{\partial x}=0, \quad 0=-\frac{\partial p_{2}}{\partial r}+\frac{1}{r} \frac{\partial}{\partial r}\left(r \frac{\partial v_{1}}{\partial r}\right)-\frac{v_{1}}{r^{2}}, \\
\operatorname{Re}\left(u_{1} \frac{\partial u_{0}}{\partial x}+u_{0} \frac{\partial u_{1}}{\partial x}+v_{2} \frac{\partial u_{0}}{\partial r}+v_{1} \frac{\partial u_{1}}{\partial r}\right) \\
=-\frac{\partial p_{2}}{\partial x}+\frac{\partial^{2} u_{0}}{\partial x^{2}}+\frac{1}{r} \frac{\partial}{\partial r}\left(r \frac{\partial u_{2}}{\partial r}\right), \\
\left.\frac{\partial u_{2}}{\partial r}\right|_{r=0}=0,\left.\quad v_{2}\right|_{r=0}=0,\left.\quad u_{2}\right|_{r=\phi(x)}=0 . \\
\left.v_{2}\right|_{r=\phi(x)}=0, \quad \int_{0}^{\phi(x)} r u_{2}(x, r) d r=0 .
\end{gathered}
$$


The definite form of the velocity and pressure gradient field will be

$$
\begin{gathered}
u(r, x)=u_{0}(r, x)+\omega u_{1}(r, x)+\omega^{2} u_{2}(r, x)+\cdots, \\
v(r, x)=\omega v_{1}(r, x)+\omega^{2} v_{2}(r, x)+\cdots, \\
\frac{\partial p}{\partial x}(r, x)=\frac{d p_{0}}{d x}(x)+\omega \frac{d p_{1}}{d x}(x)+\omega^{2} \frac{\partial p_{2}}{\partial x}(r, x)+\cdots,
\end{gathered}
$$

where the details on the solutions and the solutions to $u_{0}, u_{1}, u_{2}, v_{1}, v_{2}$, and $\frac{d p_{0}}{d x}, \frac{d p_{1}}{d x}, \frac{d p_{2}}{d x}$ are provided in Appendix A.

For the special case of regular pipe $\left(\phi=1, \phi^{\prime}=0\right)$, we obtain

$$
\begin{gathered}
u=-4 F_{0}\left(\zeta^{2}-1\right)+\omega\left[u^{*}\left(2 \zeta^{2}-1\right)+2 \operatorname{Re} v^{*} F_{0}\left(\frac{\zeta^{6}}{9}-\frac{\zeta^{+}}{2}+\frac{\zeta^{2}}{2}-\frac{1}{9}\right)\right]-\omega^{2}\left[\frac{\operatorname{Re} u^{*} v^{*}}{4}\left(\zeta^{4}-\frac{4}{3} \zeta^{2}+\frac{1}{3}\right)\right. \\
\left.+\operatorname{Re} u^{*} v^{*}\left(\frac{\zeta^{6}}{9}-\frac{\zeta^{4}}{2}+\frac{\zeta^{2}}{2}-\frac{1}{9}\right)+\operatorname{Re}^{2} v^{* 2} F_{0}\left(\frac{\zeta^{10}}{450}-\frac{\zeta^{8}}{36}+\frac{\zeta^{6}}{9}-\frac{11}{36} \zeta^{4}+\frac{38}{135} \zeta^{2}-\frac{83}{1350}\right)\right] \\
v=\omega v^{*}\left(\zeta^{3}-2 \zeta\right)-\frac{\operatorname{Re} v^{* 2}}{4}\left(\frac{\zeta^{7}}{9}-\frac{2 \zeta^{5}}{3}+\zeta^{3}-\frac{4 \zeta}{9}\right), \\
\frac{\partial p}{\partial x}=-16 F_{0}+\omega\left(8 u^{*}-12 v^{*} F_{0}\right)+\omega^{2}\left(\frac{10 \operatorname{Re} u^{*} v^{*}}{3}+\frac{88 \operatorname{Re}^{2} v^{* 2} F_{0}}{135}\right)
\end{gathered}
$$

where $z=\frac{r}{6}$. When there is no fluid exchange with the porous host rock through the walls, $u^{*}=\mathrm{v}^{*}=0$, we obtain the flow in a regular pipe: the parabolic profile for the longitudinal velocity, Fig. 4, the zero radial velocity, and the constant pressure gradient along the pipe. The fluid flow through the walls, $u^{*}=\mathrm{v}^{*}=1$, creates a non-zero radial velocity and deviates the streamlines from.

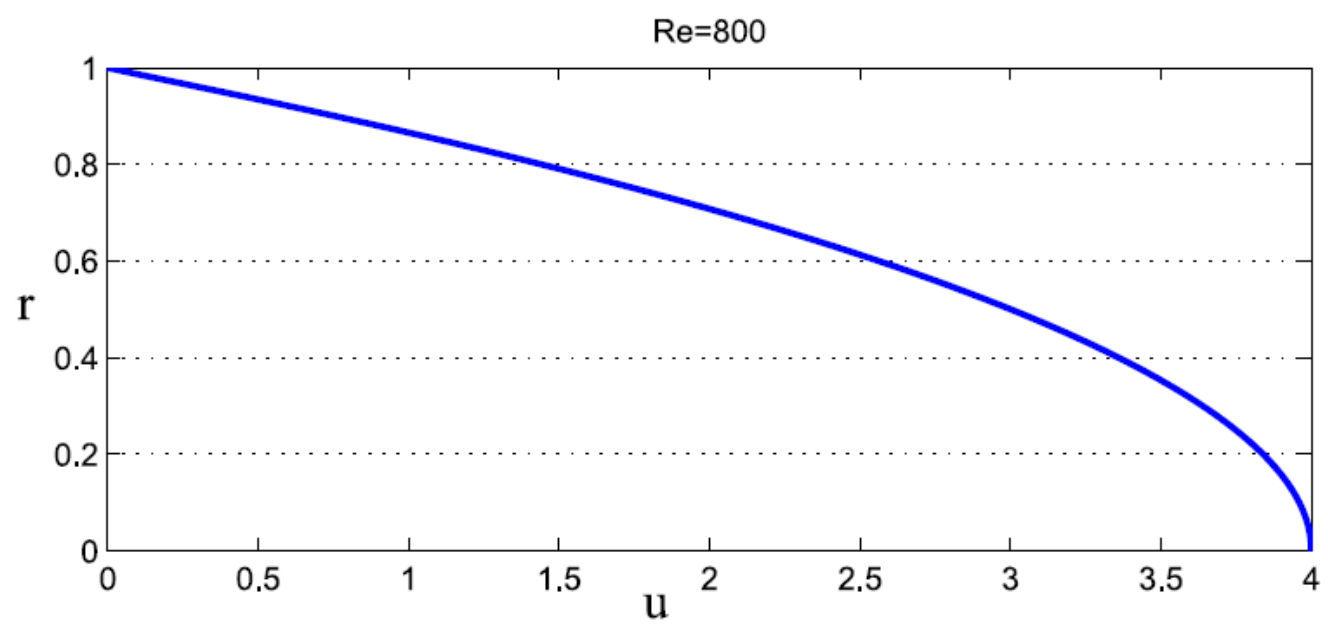

FIG. 4. Longitudinal velocity in a regular pipe with no inflow, $\mathrm{Re}=800$.

being parallel to the walls as shown in Figs. 5 and 6. At higher Reynolds, Fig. 6, a reverse flow forms in the pipe close to the wall boundaries.

For the channel with impermeable wavy walls in low Reynolds, the streamlines follow the wall geometry due to viscous effects as it is shown in Fig. 7(a). We assumed the case of the channel's sinusoidal corrugation as 


$$
\phi(x)=1+a \sin (2 \pi x / \varepsilon) .
$$

with $a$ being a dimensionless parameter equal to the ratio of the corrugation amplitude to the mean radius and $\omega=$ $0.01, \varepsilon=0.01^{0.75}=0.0316$ and $a=0.3$. The flow is from left to right, the upper half-domain is presented.

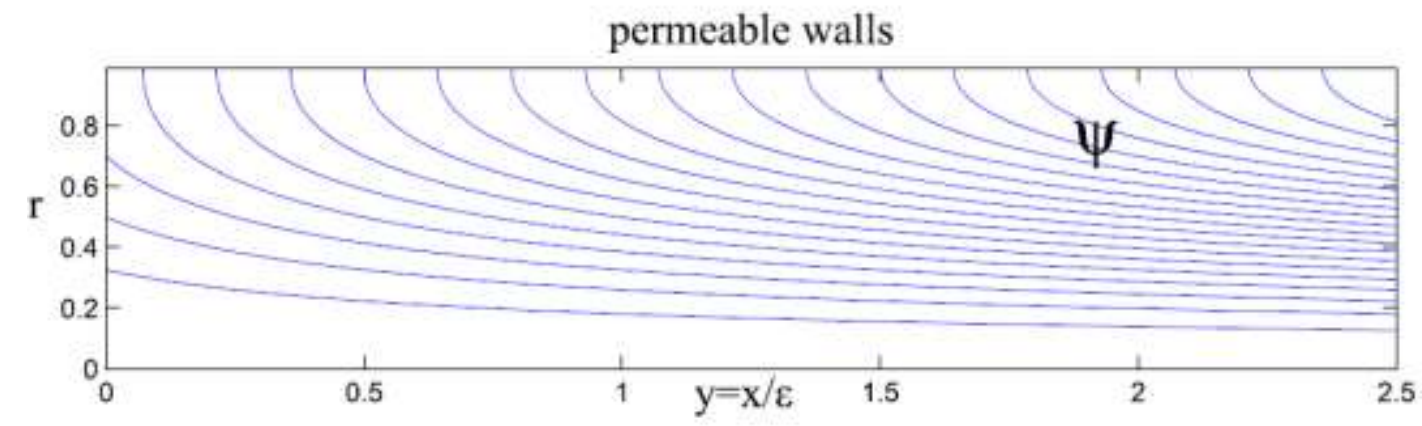

FIG. 5. Streamlines in regular pipe with inflow, $\mathrm{Re}=25$.

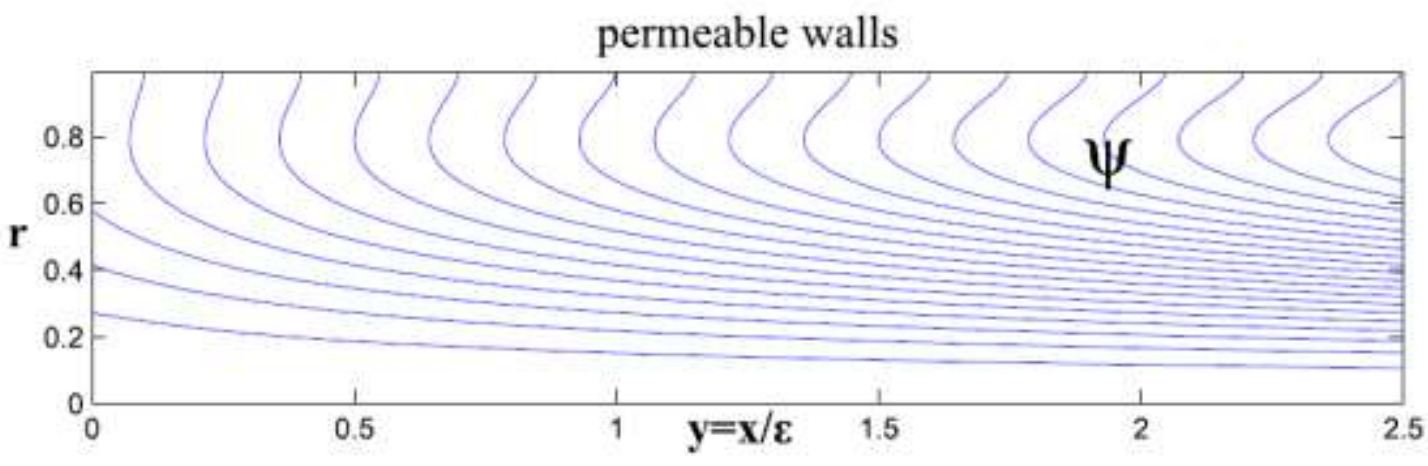

FIG. 6. Reverse flow in regular pipe with inflow, $\mathrm{Re}=35$.

\section{B. Two-scale form of the solution}

In order to average the fluctuations caused by the wavy walls, we can use the fact that the scale of the oscillations is small with respect to the long length of the channel. Due to the large difference between the two scales, the behavior at two scales may be assumed to be independent from one another. The small-scale ("fast") variable $y=$ $x / "$ is considered to be independent from the slow variable $x$. Then we introduce the extended functions $\bar{f}(r, x, y)$ so that $\left.\bar{f}(r, x, y)\right|_{y=x / \varepsilon}=f(r, x)$. The spatial derivative is defined as

$$
\frac{\partial f}{\partial x}=\frac{\partial \tilde{f}}{\partial x}+\frac{1}{\varepsilon} \frac{\partial \tilde{f}}{\partial y}
$$

The detailed two-scale results for the velocity and pressure field are given in Appendix B. For the impermeable walls, secondary flows are not visible up to some certain Reynolds number, as it is seen in Fig. 7(a). With the fluid flow through the walls secondary flows form even in low Reynolds number, Fig. 7(a). In Fig. 8, a relatively high Reynolds number, $\operatorname{Re}=5$, is chosen in order to ensure the formation of secondary flow. As it is seen in Fig. 8(a), secondary flows form even where there is no fluid flow through the walls. In Figs. 9 and 10, the stream function is plotted using the zeroth, first, and second approximations for permeable and impermeable channel walls and a relatively low Reynolds, $\operatorname{Re}=1$, and a higher Reynolds, $R e=5$. As it can be seen, at lower Reynolds the higher asymptotics for the stream function are less dominant. In higher Reynolds, the most exact correspondence with the true flow pattern is obtained in the second approximation for both cases of permeable and impermeable walls. 
It can be seen in Fig. 11 that when there is no fluid flow through the walls, the gradient of pressure along the channel is periodic. In the case of permeable walls, the pressure gradient increases along the channel axis, which results in forming the instability. The rise of instability can be noted through the appearance of the positive pressure drop which determines the reverse flow.

\section{Averaged flow model}

Let us define the single and double averaging for a two-scale function $f(r, y, x)$

$$
\langle f\rangle=\int_{0}^{1} f(r, y, x) d y, \quad\langle\langle f\rangle\rangle=\int_{0}^{1}\left(\frac{2}{\phi^{2}} \int_{0}^{\phi} f f(r, y, x) d r\right) d y .
$$

Then the averaged velocity and pressure will be

$$
\langle\langle u\rangle\rangle=2 F_{0}\left(\frac{1}{\phi^{2}}\right\rangle+\omega u^{*}\left(1-\phi(0)\left(\frac{1}{\phi^{2}}\right\rangle\right) .
$$

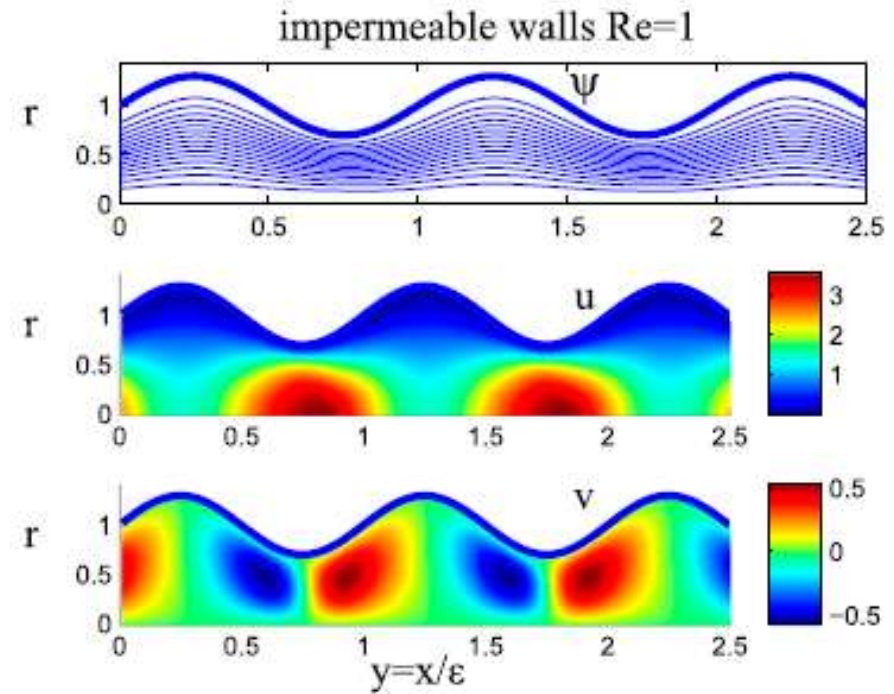

(a)
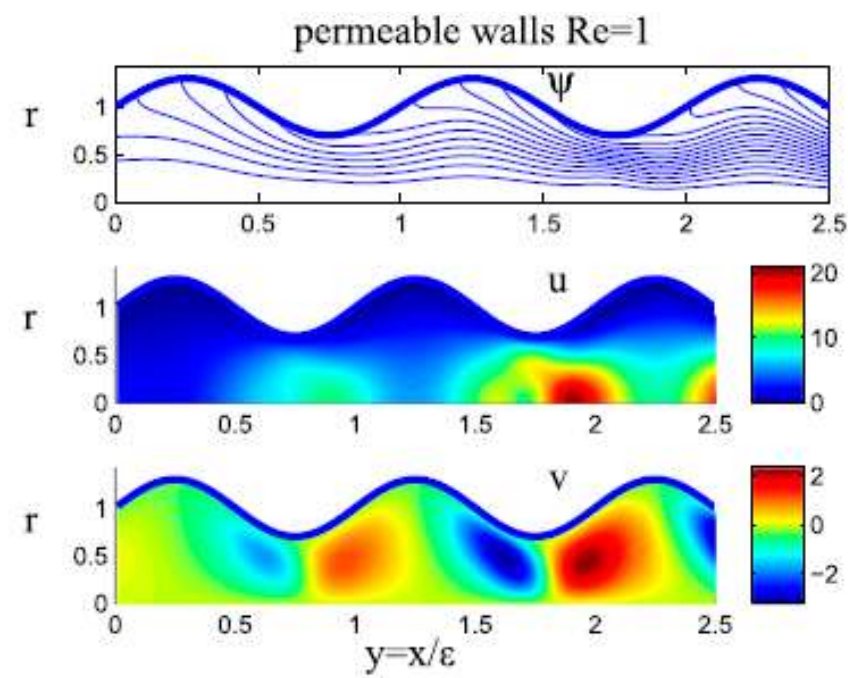

(b)

FIG. 7. Cylindrical wavy channel: Appearance of secondary flow, $\operatorname{Re}=1$ (a) without/(b) with inflow through the walls, $\phi(x)=1+a \sin (2 \pi x / \varepsilon), \omega=0.01$.

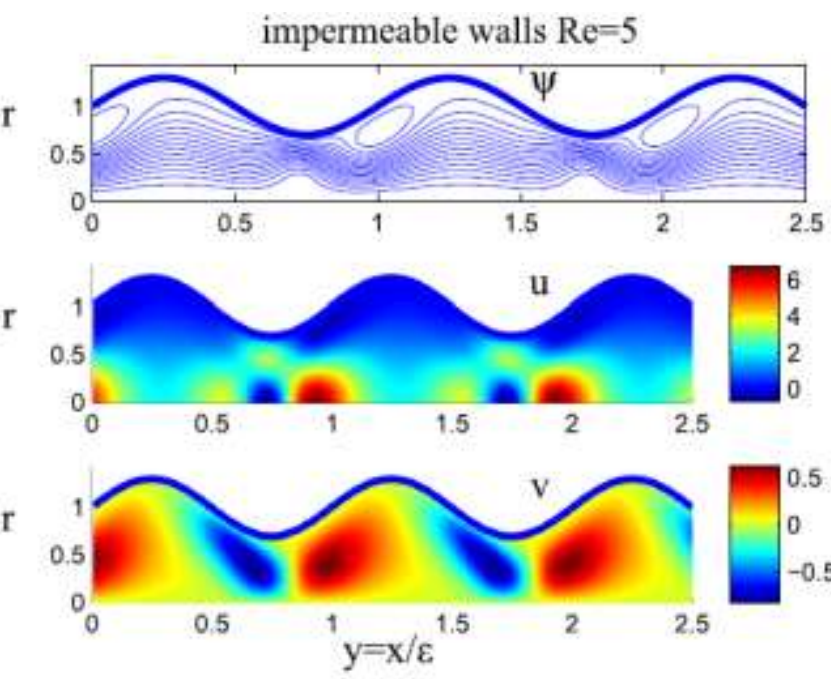

(a)

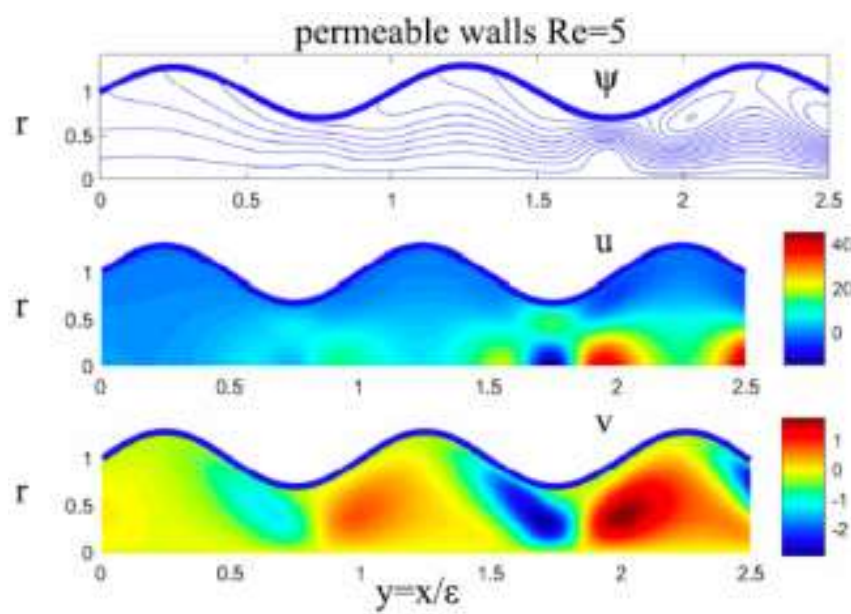

(b)

FIG. 8. Cylindrical wavy channel: Appearance of secondary flow, $\operatorname{Re}=5$ (a) without/(b) with inflow through the walls, $\phi(x)=1+a \sin (2 \pi x / \varepsilon), \omega=0.01, \varepsilon=0.01^{0.75}=0.0316$ and $a=0.3$. 


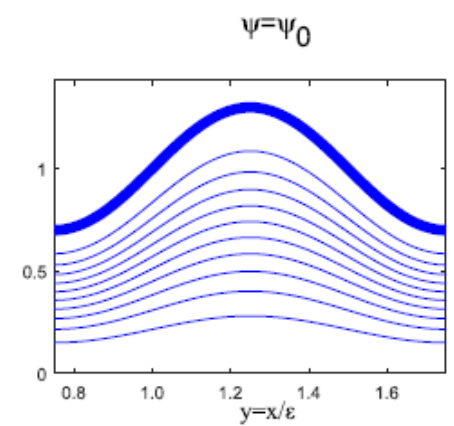

impermeable walls, $\mathrm{Re}=1$

$\psi=\psi_{0}+\omega \psi_{1}, \operatorname{Re}=1 \quad \psi=\psi_{0}+\omega \psi_{1}+\omega^{2} \psi$
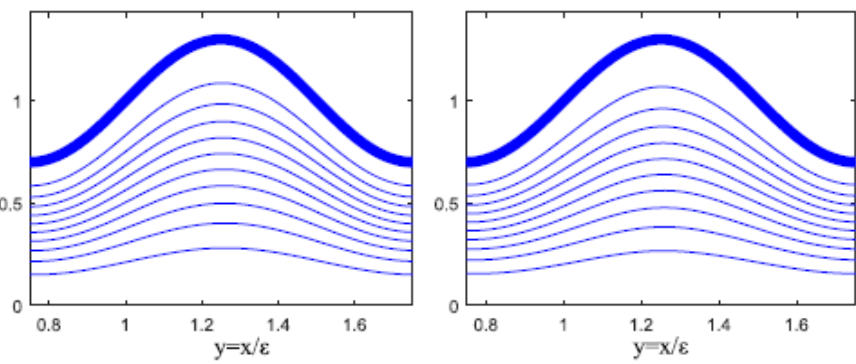

(a)

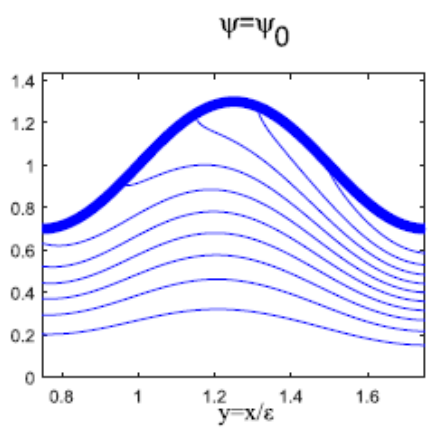

permeable walls, $\mathrm{Re}=1$ $\psi=\psi_{0}+\omega \psi_{1}$
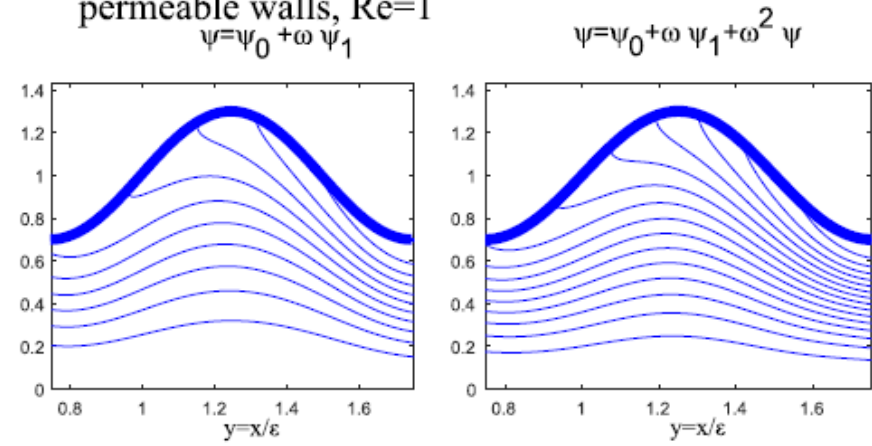

(b)

FIG. 9. Cylindrical wavy channel (impermeable walls): zero, first, and second approximation of the streamlines in the second cell of corrugation, $\operatorname{Re}=1$ (a) without/(b) with inflow through the walls.
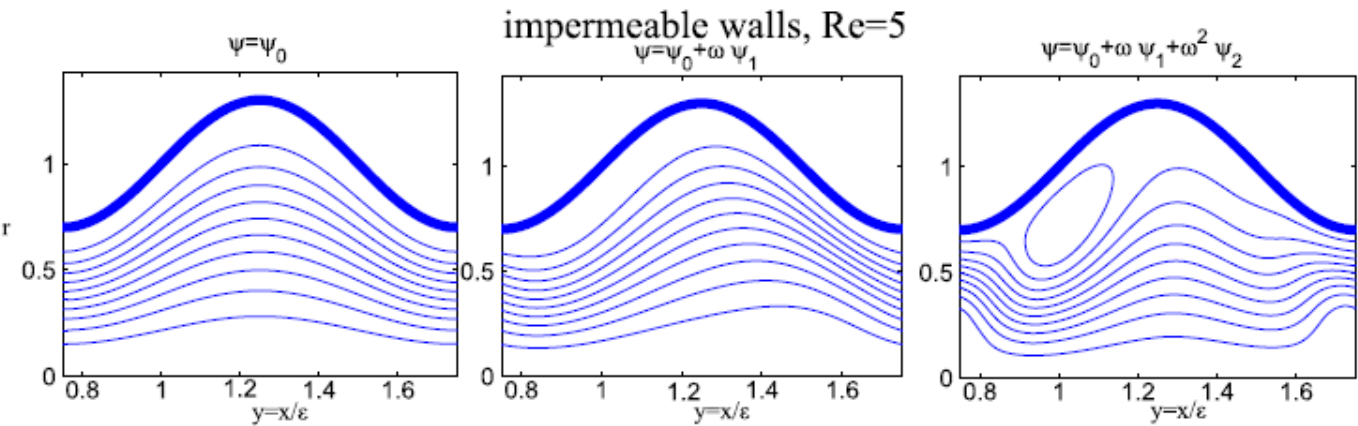

(a)
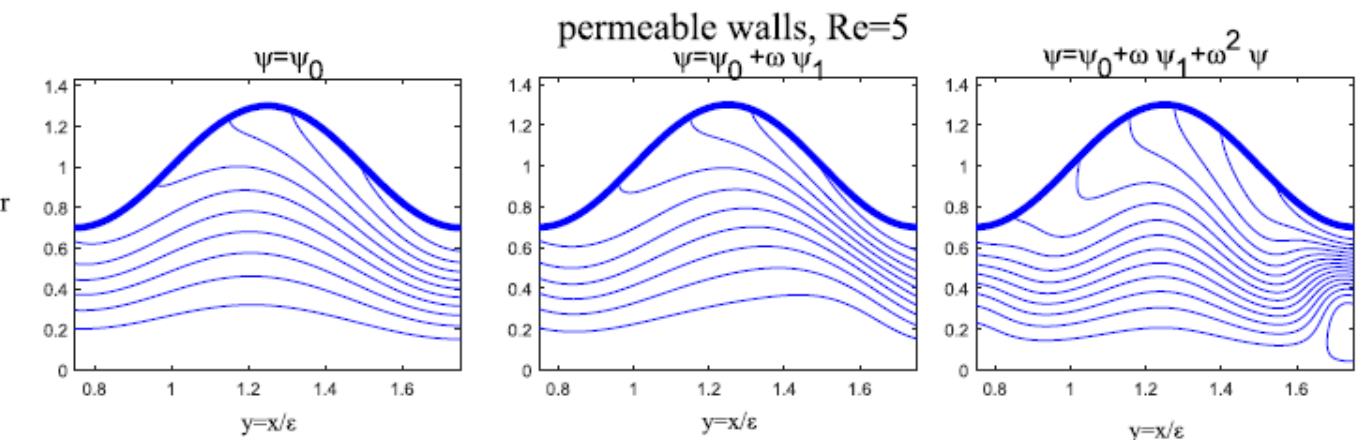

(b)

FIG. 10. Cylindrical wavy channel (impermeable walls): zero, first, and second approximation of the streamlines in the second cell of corrugation, $\operatorname{Re}=5$ (a) without/(b) with inflow through the walls. 


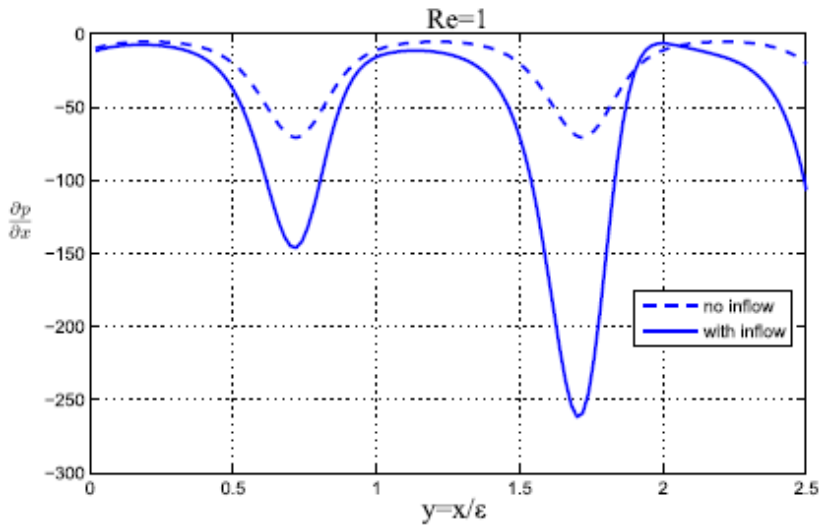

(a)

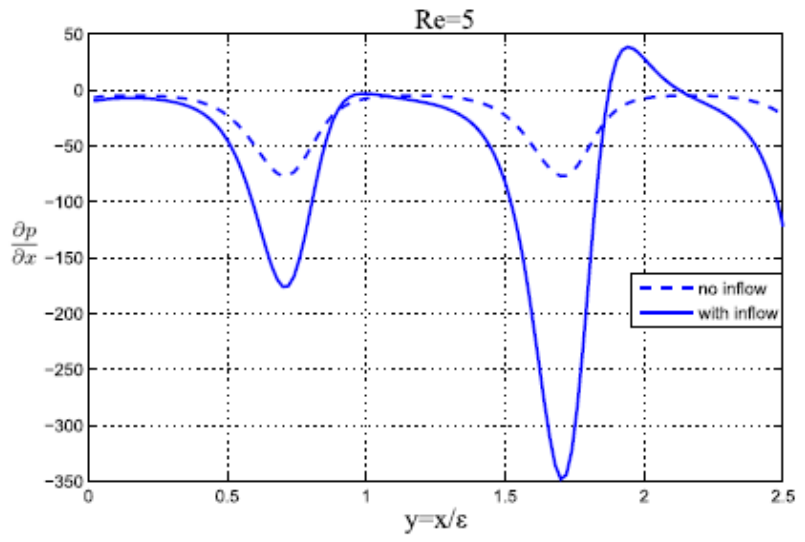

(b)

FIG. 11. Cylindrical wavy channel: The micro scale pressure drop with and without inflow for (a) $\operatorname{Re}=1$ and (b) $\operatorname{Re}=5$. (a) No instability in pressure in low Reynolds, $R e=1$. (b) Pressure instability arises in higher Reynolds, $\operatorname{Re}=5$.

$$
\langle\langle v\rangle\rangle=0,
$$

$$
\begin{aligned}
\left\langle\left(\frac{\partial p}{\partial x}\right)\right\rangle= & -16 F_{0}\left(\frac{1}{\phi^{4}}\right)+\omega\left[8 u^{*} \phi^{2}(0)\left(\frac{1}{\phi^{4}}\right)-12 \operatorname{Re} F_{0} v^{*}\left(\frac{1}{\phi^{3}}\right)\right] \\
& +\frac{\omega^{2}}{\varepsilon^{2}}\left[8 F_{0}\left(-\frac{\phi^{\prime \prime}}{\phi^{3}}+3 \frac{\phi^{\prime 2}}{\phi^{4}}\right)+\frac{8}{3} F_{0}\left(\frac{\phi^{\prime \prime}}{\phi^{3}}-\frac{7 \phi^{\prime 2}}{\phi^{4}}\right)\right. \\
& \left.-\operatorname{Re}^{2} \frac{44}{45}\left(F_{0}^{3}-1\right)\left(\frac{\phi^{\prime \prime}}{\phi^{5}}\right)+\operatorname{Re}^{2} \frac{484}{135} F_{0}^{3}\left(\frac{\phi^{\prime 2}}{\phi^{6}}\right)\right] \\
& +\omega^{2}\left[\operatorname{Re} u^{*} v^{*}\left(-\frac{8}{3 \phi}+\frac{6}{\phi^{3}}\right)+\operatorname{Re}^{2} \frac{88}{135} v^{* 2} F_{0}\left(\frac{1}{\phi^{2}}\right)\right]
\end{aligned}
$$

Returning back to the dimensional variables, and denoting $\mathcal{U} \equiv\langle\langle U\rangle\rangle, \mathcal{P} \equiv\langle\langle P\rangle\rangle$, we obtain

$$
\mathcal{U}=U^{0}\left(2 F_{0}\left\langle\frac{1}{\phi^{2}}\right\rangle+\omega u^{*}\left(1-\phi(0)\left\langle\frac{1}{\phi^{2}}\right\rangle\right)\right) .
$$

In dimensional form, the pressure drop along the channel can be said

$$
\begin{aligned}
\frac{d \mathcal{P}}{d X}= & \frac{\mu U^{0}}{R_{w}^{2}}\left\{-16 F_{0}\left(\frac{1}{\phi^{4}}\right)+8 \omega u^{*} \phi^{2}(0)\left(\frac{1}{\phi^{4}}\right)\right. \\
& \left.+\frac{\omega^{2}}{\varepsilon^{2}}\left(8 F_{0}\left(-\frac{\phi^{\prime \prime}}{\phi^{3}}+3 \frac{\phi^{2}}{\phi^{4}}\right)+\frac{8}{3} F_{0}\left(\frac{\phi^{\prime \prime}}{\phi^{3}}-\frac{7 \phi^{\prime 2}}{\phi^{4}}\right)\right)\right\} \\
& +\frac{\rho U^{02}}{R_{w}}\left\{-12 \omega F_{0} v^{*}\left(\frac{1}{\phi^{3}}\right)+\omega^{2} u^{*} v^{*}\left(\frac{6}{\phi^{3}}-\frac{8}{3 \phi}\right)\right\} \\
& +\frac{\rho^{2} U^{0^{2}}}{\mu} \frac{44}{45}\left\{\omega^{2} \frac{2}{3} F_{0} v^{* 2}\left(\frac{1}{\phi^{2}}\right)\right. \\
& \left.+\frac{\omega^{2}}{\varepsilon^{2}}\left(-\left(\frac{\phi^{\prime \prime}}{\phi^{5}}\right)\left(1-F_{0}^{3}\right)+\frac{11}{3} F_{0}^{3}\left(\frac{\phi^{2}}{\phi^{6}}\right)\right)\right\}
\end{aligned}
$$

Replacing $U^{0}$ by $\mathrm{U}$ by using (35), we obtain the averaged momentum balance equation in the form of a cubic law 


$$
-\frac{d \mathcal{P}}{d X}=\frac{\mu \mathcal{U}}{K^{\mathrm{ef}}}+\beta^{\text {ef }} \rho \mathcal{U}^{2}+\frac{\gamma^{\text {ef }} \rho^{2} \mathcal{U}^{\beta}}{\mu}
$$

where

$$
\begin{gathered}
K^{e f}=\frac{R_{w}^{2}}{8} \lambda, \\
\lambda=\frac{2 F_{0}\left(\frac{1}{\phi^{2}}\right\rangle+\omega u^{*}\left(1-\phi(0)\left\langle\frac{1}{\phi^{2}}\right\rangle\right)}{2 F_{0}\left\langle\frac{1}{\phi^{4}}\right\rangle-\omega u^{*} \phi^{2}(0)\left\langle\frac{1}{\phi^{4}}\right\rangle+\frac{2}{3} \frac{\omega^{2}}{\varepsilon^{2}} F_{0}\left\langle\frac{\phi^{\prime \prime}}{\phi^{3}}-\frac{\phi^{\prime 2}}{\phi^{4}}\right\rangle},(24) \\
\beta^{e f}=\frac{v^{*} \omega}{R_{w}} \frac{12 F_{0}\left\langle\frac{1}{\phi^{3}}\right\rangle+\omega u^{*}\left(\frac{8}{3 \phi}-\frac{6}{\phi^{3}}\right\rangle}{\left(2 F_{0}\left\langle\frac{1}{\phi^{2}}\right\rangle+\omega u^{*}\left(1-\phi(0)\left\langle\frac{1}{\phi^{2}}\right)\right)\right)^{2}}, \\
\gamma^{e f}=-\frac{44 \omega^{2}}{135} \frac{2 F_{0} v^{* 2}\left\langle\frac{1}{\phi^{2}}\right\rangle+\frac{1}{\varepsilon^{2}}\left(-3\left\langle\frac{\phi^{\prime \prime}}{\phi^{5}}\right\rangle\left(1-F_{0}^{3}\right)+11 F_{0}^{3}\left\langle\frac{\phi^{\prime 2}}{\phi^{6}}\right\rangle\right)}{\left(2 F_{0}\left\langle\frac{1}{\phi^{2}}\right\rangle+\omega u^{*}\left(1-\phi(0)\left\langle\frac{1}{\phi^{2}}\right\rangle\right)\right)^{3}} .
\end{gathered}
$$

Three effective parameters determine the averaged behaviour of the system:

- $K^{\text {ef }}$ is the effective permeability $\left[\mathrm{m}^{2}\right]$, the parameter responsible for the pure viscous dissipation (the friction) which corresponds to Darcy's law. The effective permeability also includes the consequences of irregular boundaries as the local Darcy like term. These effects are assumed as a correction to the effective permeability, here determined in terms of coefficients of $\omega^{2} / \varepsilon^{2}$ in parameter $\lambda$, the corrugation parameter. In Fig. 12 the corrugation parameter is plotted vs. the oscillation amplitude for both permeable walls and impermeable walls. It can be seen that the presence of irregularities in the geometry of the boundaries decreases the effective permeability of the channel significantly, while the presence or absence of fluid flow through the walls has not a significant role on the effective permeability.

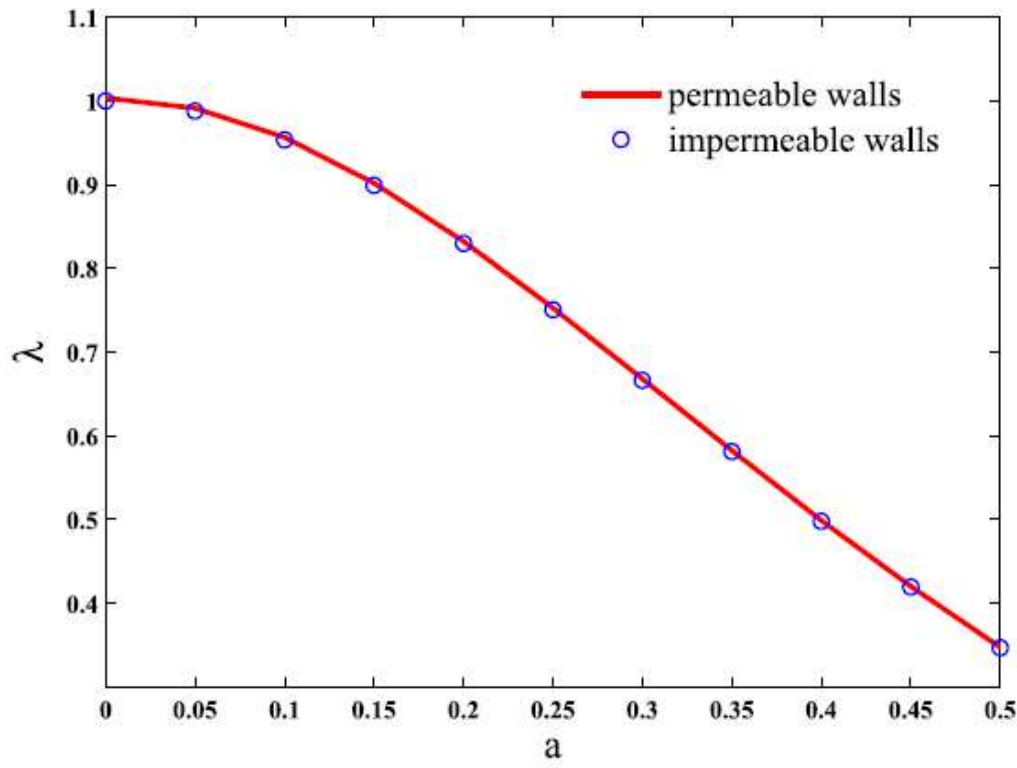

FIG. 12. Cylindrical wavy channel: Pure viscous effects coefficient (effective permeability) vs. different corrugation amplitudes a, $\omega=0.01, \varepsilon=0.01^{0.75}$. 
- $\beta^{\text {ef }}$ is the inertia parameter having the dimension $\left[m^{-1}\right]$. It is responsible for the integral losses of the kinetic energy within a period caused by the radial component of the inflow velocity which is orthogonal to the channel's axis, $\mathrm{v}^{*}$. The radial component of inflow deviates fluid from the axial direction of flow, which reduces the fluid axial velocity. The energy transferred with the inflow plays the role of a decelerator, which means the appearance of a negative acceleration, or the inertia losses of energy. $\beta^{\text {ef }}$ vanishes in the absence of fluid exchange through the walls as shown in Fig. 13.

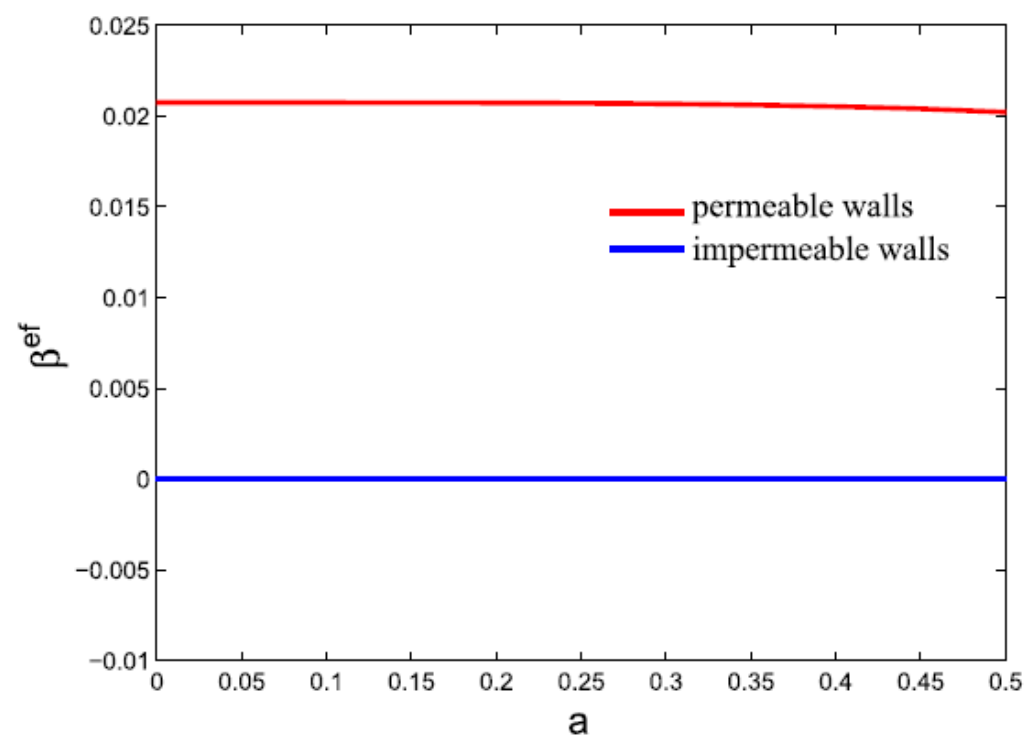

FIG. 13. Cylindrical wavy channel: Pure inertial effect coefficient vs. different corrugation amplitudes a, $\omega=0.01, \varepsilon=0.01^{0.75}$.

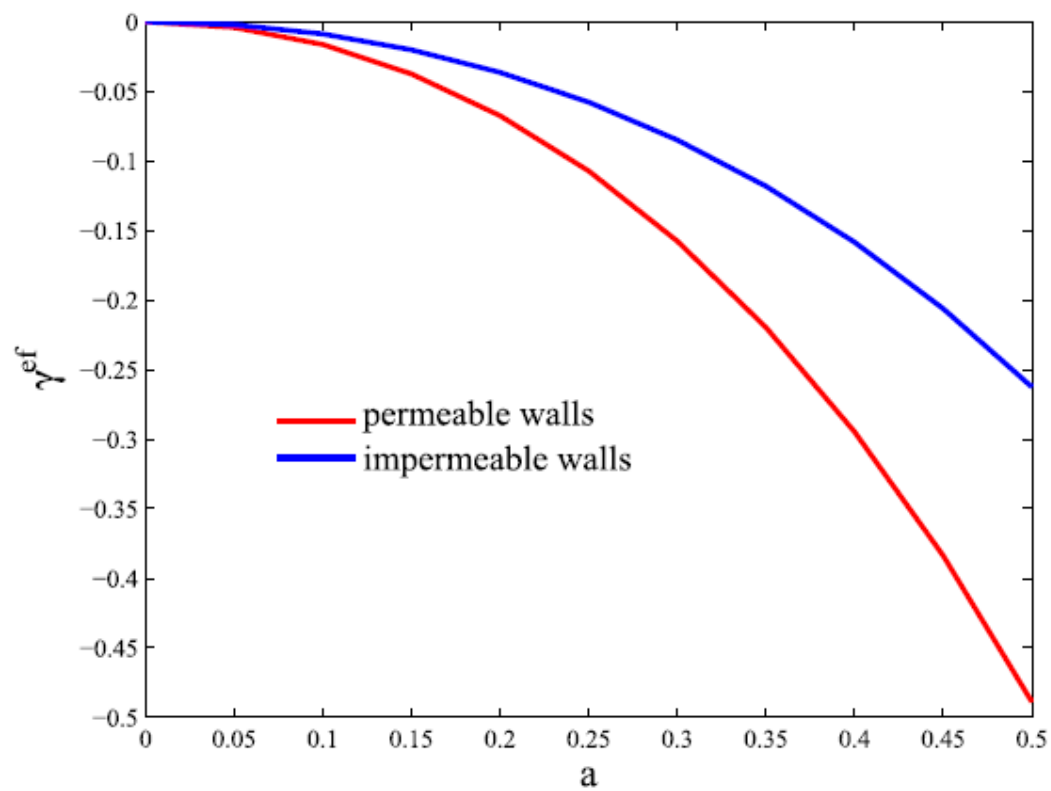

FIG. 14. Cylindrical wavy channel: Cross visco-inertial effect coefficient vs. different corrugation amplitudes a, $\omega=0.01, \varepsilon=0.01^{0.75}$.

with the inflow plays the role of a decelerator, which means the appearance of a negative acceleration, or the inertia losses of energy. _ef vanishes in the absence of fluid exchange through the walls as shown in Fig. 13.

- $\quad \gamma^{\text {ef }}$ is the dimensionless parameter responsible for the visco-inertial cross-effect that represents a supplementary viscous dissipation produced by the deviation of the streamlines due to increase in Reynolds. As the streamline deformation is caused by the inertial forces, this term depends on Re. $\gamma^{\text {ef }}$ represents the global and local cross visco-inertial effect. The global effect is zero in the absence of fluid flow through the walls. As it is shown in Fig. 14, this term vanishes for a regular pipe. 
In the special case of a regular pipe with permeable walls, one will have $F_{0}=1 / 2+\mathrm{v}^{*} \mathrm{x}$ and

$$
\begin{gathered}
K^{e f f}=\frac{R_{w}^{2}}{8} \lambda, \quad \lambda=\frac{2 F_{0}}{2 F_{0}-u^{*} \omega}, \\
\beta^{e f f}=\frac{3 \omega F_{0} v^{*}-\frac{5}{6} \omega^{2} u^{*} v^{*}}{F_{0}^{2}}, \\
\gamma^{e f f}=-\frac{11 \omega^{2}}{135} \frac{v^{* 2}}{F_{0}^{2}} .
\end{gathered}
$$

It is seen that for a pipe with permeable walls, the pure inertia effects and cross visco-inertial effects enter the averaged pressure drop. For a regular pipe with no inflow, we reduce to Hagen-Poiseuille equation for pressure drop in pipes, with $\lambda=1$. Also in the presence of fluid flow through the walls, the effective permeability increases for a regular pipe, Fig. 15. As a result of $\omega<<1$, the limit value of the effective permeability reduces to the one for the pipe with impermeable walls.

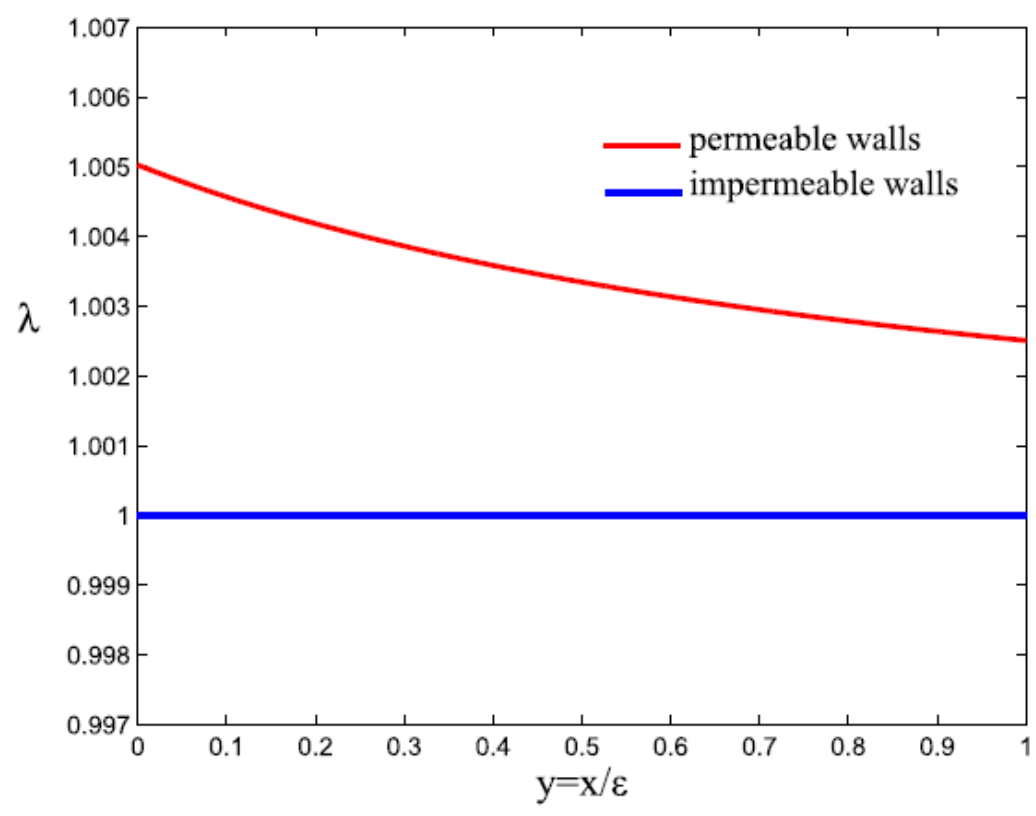

FIG. 15. Cylindrical wavy channel: Effective permeability of a regular pipe with/without inflow through the walls.

\section{PARALLEL-PLATE WAVY CHANNELS}

The same approach presented in Sec. II can be applied to investigate the flow in a slab-shaped parallel-plate wavy channel with permeablewalls immersed in the porous medium, as shown in Fig. 16. The channel's depth perpendicular to the direction of the flow is infinite, so the problem is assumed to be 2D, Fig. 17.

The mean channel's aperture is $H$ and the characteristic length of the channel is $L$. The upper wall is described in $(X, Y, Z)$ coordinates as $\mathrm{W}(X, Z)=\{0<X<L, 0<Z<H \phi(X)\}$, where $\phi(X)$ is the periodic function with period $l$, the mean value $1 / 2$, and $\phi(0)=1 / 2$. In case when $\phi(X)=1 / 2$, the channel walls are planes. The boundary condition at the inlet section imposes the given mean velocity $U^{0}$ as

$$
U^{\circ} \equiv \frac{1}{H \phi(0)} \int_{0}^{H \phi(0)} U_{X}(0, Z) d Z
$$


where $U_{X}$ is the longitudinal component of the fluid velocity. The influx from porous host rock is assumed to be uniform and equal to $\left(U_{X}^{*}, U_{Z}{ }^{*}\right)$. The following dimensionless variables:

$$
x=\frac{X}{L}, \quad z=\frac{Z}{H}, \quad u=\frac{U_{X}}{U^{\circ}}, \quad v=\frac{U_{Z}}{U^{\circ}}, \quad p=\frac{P}{p^{\circ}},
$$

will be used with $p^{\circ}=\frac{\mu U^{\circ} L}{H^{2}}$ and $R e=\frac{\rho U^{\circ} H}{\mu}$.

The steady-state mass conservation and Navier-Stokes equations for the incompressible fluid flow in the domain $W(x, z)=\{0<x<1,0<z<\phi(x)\}$ will rewrite to

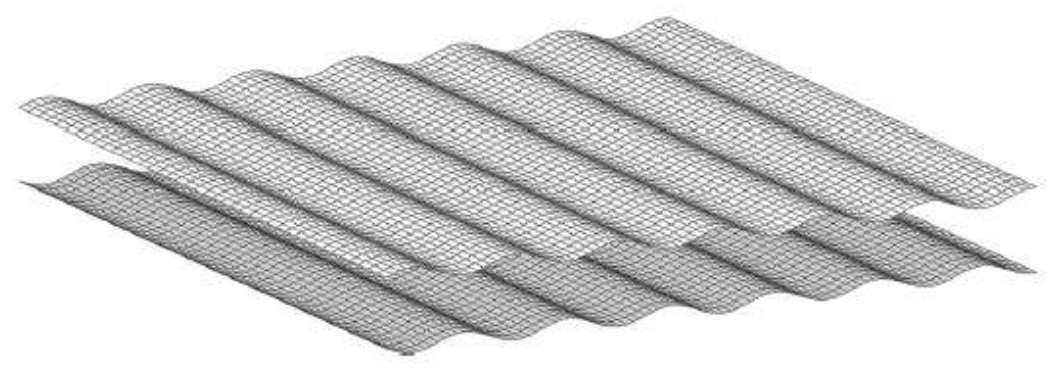

FIG. 16. Parallel-plate channel with wavy walls.

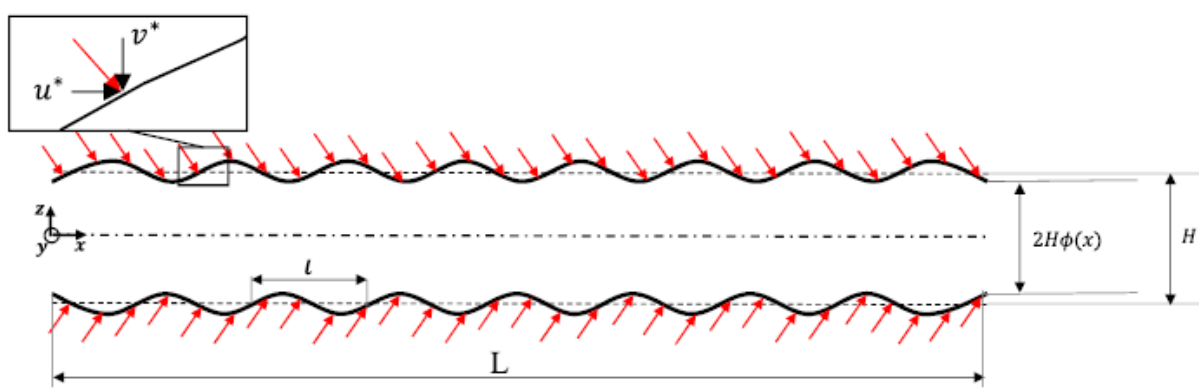

FIG. 17. Parallel-plate wavy channel with uniform fluid flow through the walls.

$$
\begin{gathered}
\omega \frac{\partial u}{\partial x}+\frac{\partial v}{\partial z}=0 \\
\operatorname{Re}\left(\omega u \frac{\partial u}{\partial x}+v \frac{\partial u}{\partial z}\right)=-\frac{\partial p}{\partial x}+\omega^{2} \frac{\partial^{2} u}{\partial x^{2}}+\frac{\partial^{2} u}{\partial z^{2}} \\
\operatorname{Re}\left(\omega u \frac{\partial v}{\partial x}+v \frac{\partial v}{\partial z}\right)=-\frac{1}{\omega} \frac{\partial p}{\partial z}+\omega^{2} \frac{\partial^{2} v}{\partial x^{2}}+\frac{\partial^{2} v}{\partial z^{2}} .
\end{gathered}
$$

The dimensionless boundary conditions on the channel's inlet and wall boundaries will be

$$
\begin{gathered}
\frac{1}{2}=\int_{0}^{\phi(0)} u(0, z) d z=\int_{0}^{1 / 2} u(0, z) d z, \\
\left.v\right|_{z=\phi(x)}=-\omega v^{*},\left.\quad u\right|_{z=\phi(x)}=\omega u^{*}, \\
\left.\frac{\partial u}{\partial z}\right|_{z=0}=0,\left.\quad v\right|_{z=0}=0 .
\end{gathered}
$$




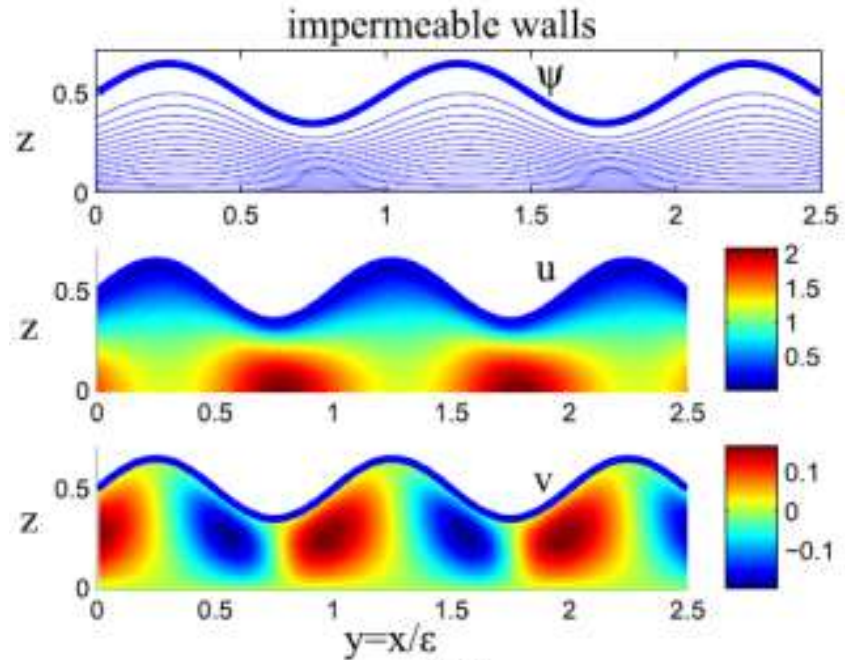

(a)

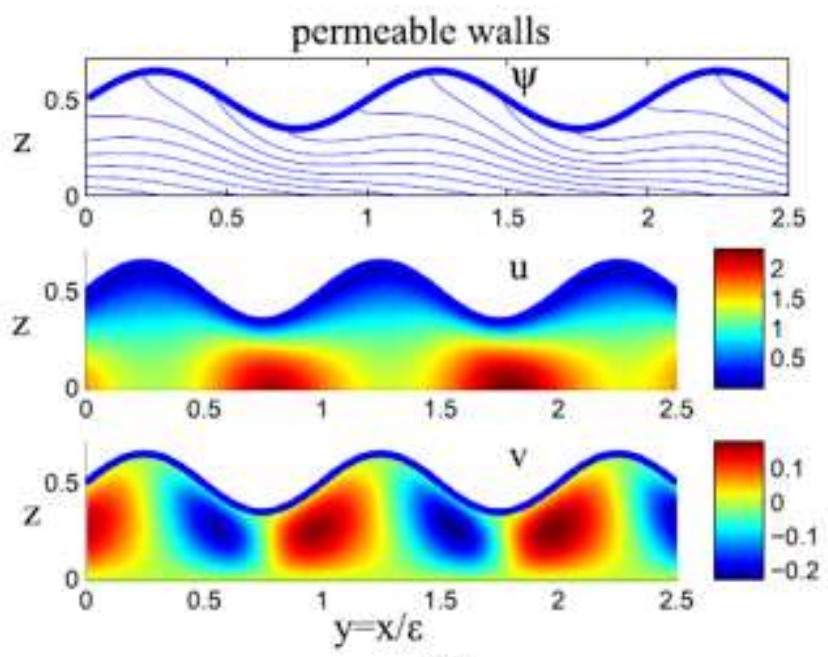

(b)

FIG. 18. Parallel-plate wavy channel: Stream function $\psi$, longitudinal velocity $u$, and transversal velocity $\mathrm{v}$ for $\operatorname{Re}=12$, wall geometry $\phi(x)=0.5(1+a \sin (2 \pi x / \varepsilon))$ for $\omega=0.01, \varepsilon=0.01^{0.75}=0.0316, a=0.3$. (a) Impermeable walls (b) Permeable walls.

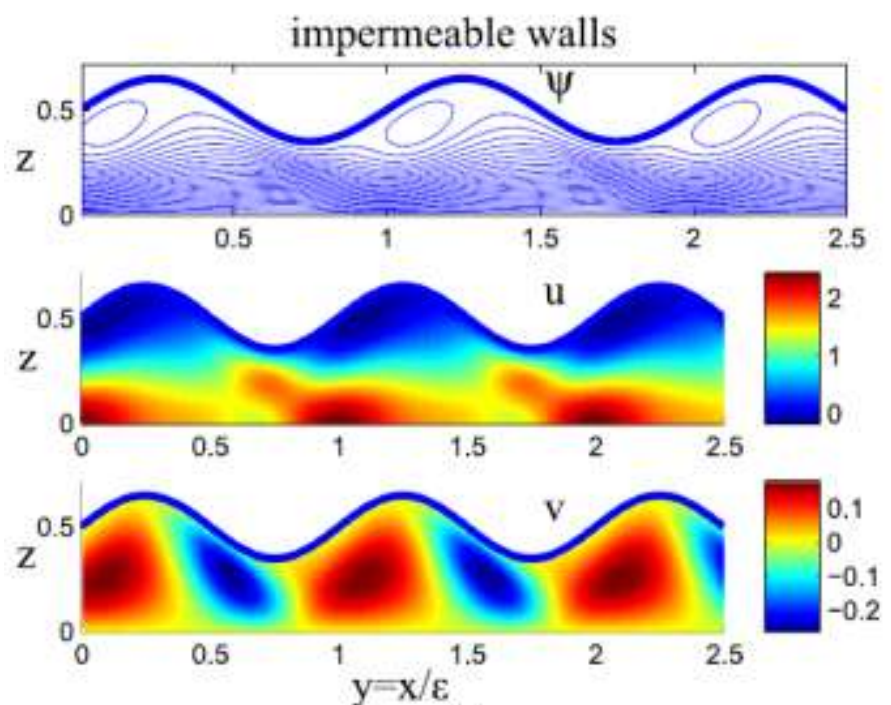

(a)

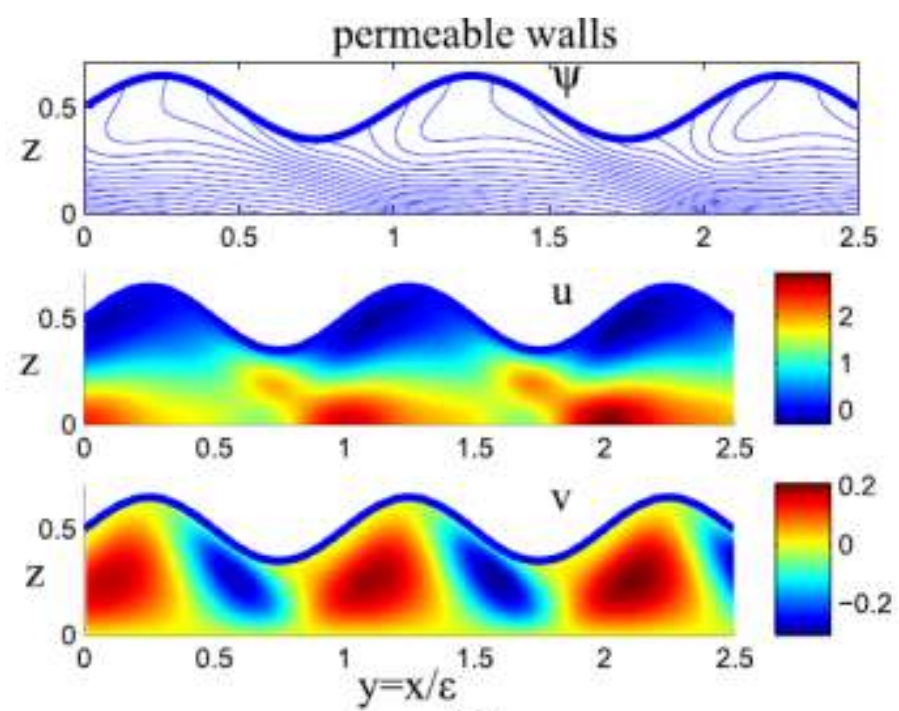

(b)

FIG. 19. Parallel-plate wavy channel: Stream function $\psi$, longitudinal velocity $u$, and transversal velocity v for $\operatorname{Re}=55$, wall geometry $\phi(x)=0.5(1+a \sin (2 \pi x / \varepsilon))$ for $\omega=0.01, \varepsilon=0.01^{0.75}=0.0316, a=0.3$. (a) Impermeable walls (b) Permeable walls.

$$
\begin{gathered}
\int_{0}^{\phi(x)} u(x, z) d z=F_{0}(x)+\omega F_{1}(x), \\
F_{0}(x) \equiv \frac{1}{2}+\int_{0}^{x} v^{*} d \bar{x}, \\
F_{1}(x) \equiv u^{*}(\phi(x)-\phi(0)) .
\end{gathered}
$$

Introducing $\zeta=\frac{z}{\phi}$ and the asymptotic expansion in terms of powers of $\omega$, the solution in the two-scale form using the small scale variable $y=x / \varepsilon$ will be obtained as given in Appendix C.

In Fig. 18, the streamlines and pressure field are plotted for a parallel-plate wavy channel with walls of geometry 
$\phi(x)=0.5(1+a \sin (2 \pi x / \varepsilon))$ where $a$ is the ratio of the wavy amplitude at half of the mean aperture. In this case, $\omega=0.01, \varepsilon=0.01^{0.75}, a=0.3$, and $\operatorname{Re}=12$. It is seen that at small $\operatorname{Re}$, the fluid flow through the walls, Fig. 18(b), influences the streamlines in the vicinity of walls, but weakly influences the velocity magnitude.

In Fig. 19, the same plots are presented for a relatively higher Reynolds $\operatorname{Re}=55$. For higher Reynolds number, the flow through the walls has a significant impact on streamlines and the velocity field in the channel. In the case of permeable walls, the flow in the channel is not periodic anymore.

The pressure gradient along the channel for $\mathrm{Re}=12$ and $\mathrm{Re}=20$ is presented in Fig. 20. The pressure drop along the channel is periodic when there is no fluid flow through the channel walls. In the case of permeable walls, the amplitude of oscillations of the pressure gradient increases along the flow direction. At high Reynolds, starting around $\operatorname{Re}=20$, the pressure gradient becomes locally positive which means the appearance of a local reverse flow. This counter-current flow might result in the appearance of the instability.

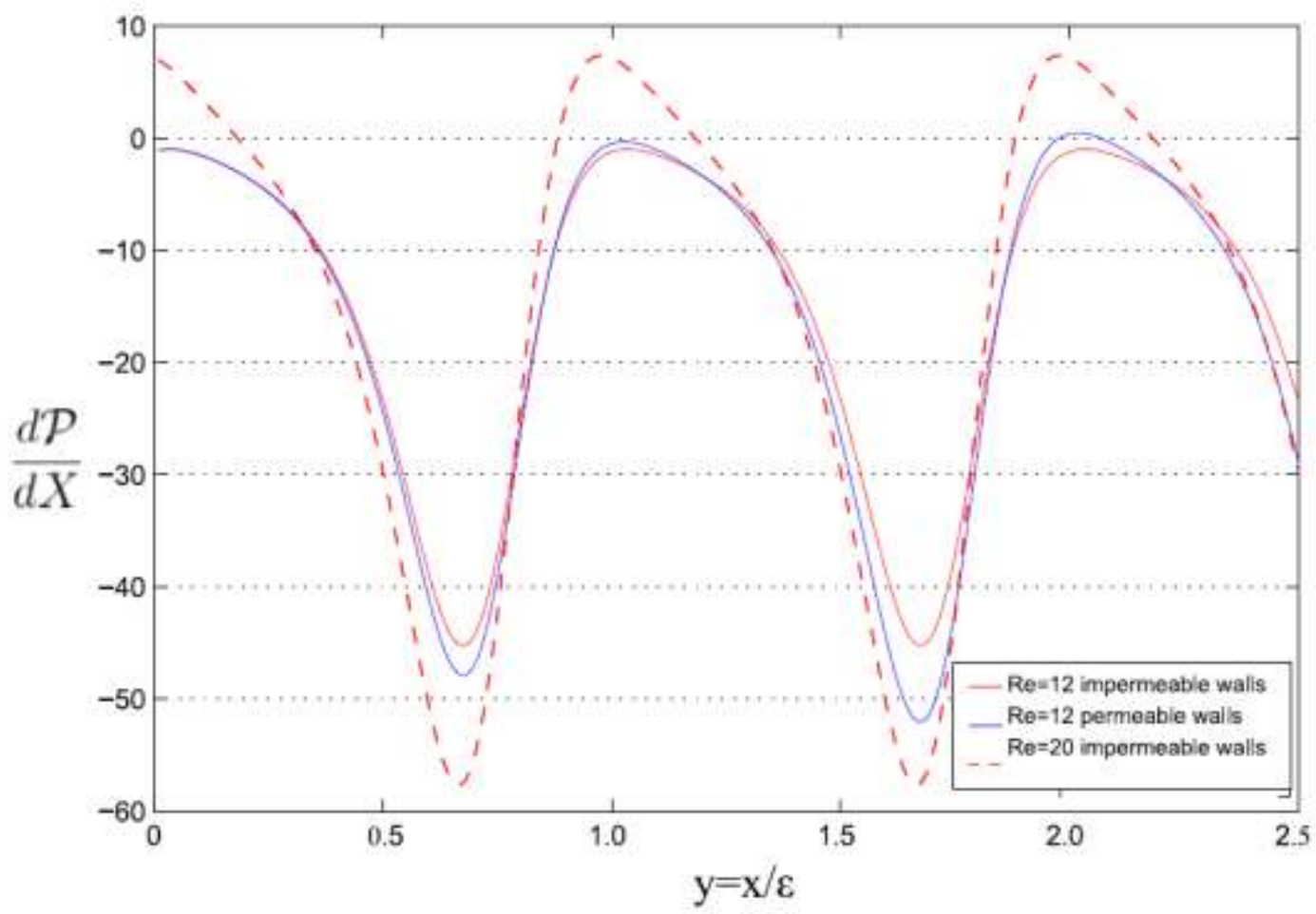

FIG. 20. Parallel-plate wavy channel: Pressure drop $d p / d x$ averaged over aperture of channel, $\omega=0.01$, $\varepsilon=0.01^{0.75}=0.0316$.

We apply twice averaging defined as

$$
\langle\langle f\rangle\rangle=\int_{0}^{1}\left(\frac{1}{\phi(y)} \int_{0}^{\phi(y)} \tilde{f}(x, y, z) d z\right) d y,
$$

which gives the averaged longitudinal velocity $\mathrm{C} 1$, transversal velocity $\mathrm{C} 2$, and pressure gradient along the channel's length $\mathrm{C} 3$ in the dimensional form $\mathcal{U} \equiv\langle\langle U\rangle\rangle, \bar{V} \equiv\langle\langle V\rangle\rangle, \mathcal{P} \equiv\langle\langle\vec{P}\rangle\rangle$, as the following:

$$
\begin{gathered}
\mathcal{U}=U^{0}\left(F_{0}\left\langle\frac{1}{\phi}\right\rangle+\omega u^{*}\left(1-\phi(0)\left\langle\frac{1}{\phi}\right\rangle\right)\right), \\
\mathcal{V}=0,
\end{gathered}
$$




$$
\begin{aligned}
\frac{d \mathcal{P}}{d X}= & \frac{\mu U^{0}}{H^{2}}\left\{-3 F_{0}\left(\frac{1}{\phi^{3}}\right)+\omega 3 u^{*} \phi(0)\left(\frac{1}{\phi^{3}}\right)+\frac{\omega^{2}}{\varepsilon^{2}} \frac{3}{5} F_{0}\left(\left\langle\frac{\left(\phi_{y}^{\prime}\right)^{2}}{\phi^{3}}\right\rangle-2\left(\frac{\phi_{y y}^{\prime \prime}}{\phi^{2}}\right)\right)\right\}+\frac{\rho U^{0} v^{*}}{H}\left\{-\omega \frac{81}{35} F_{0}\left(\frac{1}{\phi^{2}}\right\rangle\right. \\
& \left.+\omega^{2}\left[-\frac{6}{5} u^{*}\left(\frac{1}{\phi}\right\rangle+\frac{81}{35} u^{*} \phi(0)\left\langle\frac{1}{\phi^{2}}\right)\right]\right\}+\frac{\rho^{2} U^{0^{3}}}{\mu} \frac{3}{13475}\left\{\frac{\omega^{2}}{\varepsilon^{2}} 4 F_{0}^{3}\left(-39\left(\frac{\phi_{y y}^{\prime \prime}}{\phi^{2}}\right)+52\left(\frac{\phi_{y}^{\prime 2}}{\phi^{3}}\right\rangle\right)-\omega^{2} 78 F_{0} v^{-2}\left\langle\frac{1}{\phi}\right\rangle\right\} .
\end{aligned}
$$

Replacing $U^{0}$ by $U$ by using (35), we obtain the averaged momentum balance equation in the form of a cubic law

$$
-\frac{d \mathcal{P}}{d X}=\frac{\mu \mathcal{U}}{K^{\mathrm{ef}}}+\beta^{\mathrm{ef}} \rho \mathcal{U}^{2}+\frac{\gamma^{\mathrm{ef}} \rho^{2} \mathcal{U}^{3}}{\mu}
$$

where

$$
\begin{aligned}
& K^{\mathrm{ef}} \equiv \frac{H^{2}}{12} \lambda \text {. } \\
& \lambda=\frac{4\left[F_{0}\left\langle\frac{1}{\phi}\right\rangle+\omega u^{*}\left(1-\phi(0)\left\langle\frac{1}{\phi}\right\rangle\right)\right]}{F_{0}\left\langle\frac{1}{\phi^{3}}\right\rangle-\omega u^{*} \phi(0)\left\langle\frac{1}{\phi^{3}}\right\rangle-\frac{\omega^{2}}{\varepsilon^{2}} \frac{F_{0}}{5}\left(\left\langle\frac{\left(\phi_{y}^{\prime}\right)^{2}}{\phi^{3}}\right\rangle-2\left\langle\frac{\phi_{y y}^{\prime \prime}}{\phi^{2}}\right\rangle\right)} \\
& \beta^{\mathrm{ef}} \equiv \frac{v^{*} \omega}{35 H} \frac{81 F_{0}\left(\frac{1}{\phi^{2}}\right)+\omega u^{*}\left[42\left(\frac{1}{\phi}\right)-81 \phi(0)\left(\frac{1}{\phi^{2}}\right)\right]}{\left(F_{0}\left(\frac{1}{\phi}\right)+\omega u^{*}\left(1-\phi(0)\left(\frac{1}{\phi}\right)\right)\right)^{2}} \\
& \gamma^{\mathrm{ef}} \equiv \frac{6}{13475} \frac{\frac{\omega^{2}}{\varepsilon^{2}} 2 F_{0}^{3}\left(39\left(\frac{\phi_{y y}^{\prime \prime}}{\phi^{2}}\right)-52\left(\frac{\phi_{y}^{\prime 2}}{\phi^{3}}\right)\right)+\omega^{2} 39 F_{0} v^{* 2}\left\langle\frac{1}{\phi}\right\rangle}{\left(F_{0}\left(\frac{1}{\phi}\right)+\omega u^{*}\left(1-\phi(0)\left(\frac{1}{\phi}\right)\right)\right)^{3}} .
\end{aligned}
$$

The effective permeability $K^{\text {ef }}[\mathrm{m} 2]$ depends on the geometry of the channel and weakly depends on the influx through the walls.

The inertial term $\beta^{\mathrm{ef}}\left[\mathrm{m}^{-1}\right]$ is responsible for the integral losses of the kinetic energy caused by the component of the inflow velocity which is orthogonal to the channel's axis, $\mathrm{v}^{*}$.

$\gamma^{\text {ef }}$ is the dimensionless parameter responsible for the visco-inertial cross-effect.

Two limit cases help to analyze the behavior of these parameters: the non-corrugated channel and the impermeable walls.

For the case of non-corrugated walls, when $\phi=1 / 2$, one has

$$
\begin{gathered}
K^{\mathrm{ef}} \equiv \frac{H^{2}}{12} \lambda, \\
\lambda=\frac{2 F_{0}}{2 F_{0}-\omega u^{*}},
\end{gathered}
$$




$$
\begin{gathered}
\beta^{\mathrm{ef}} \equiv \frac{v^{*} \omega}{35 H} \frac{81 F_{0}-19.5 \omega u^{*}}{F_{0}^{2}} . \\
\gamma^{\mathrm{ef}} \equiv \frac{117 \omega^{2} v^{* 2}}{26950 F_{0}^{2}} .
\end{gathered}
$$

For the case of impermeable walls $u^{*}=\mathrm{v}^{*}=0$, one writes

$$
\begin{gathered}
K^{\mathrm{ef}} \equiv \frac{H^{2}}{12} \lambda, \\
\lambda=\frac{4\left(\frac{1}{\phi}\right)}{\left\langle\frac{1}{\phi^{3}}\right\rangle-\frac{\omega^{2}}{\varepsilon^{2}} \frac{1}{5}\left(\left\langle\frac{\left(\phi_{y}^{\prime}\right)^{2}}{\phi^{3}}\right\rangle-2\left\langle\frac{\phi_{y y}^{\prime \prime}}{\phi^{2}}\right\rangle\right)},
\end{gathered}
$$

The mass balance along the channel possesses the following integral relation:

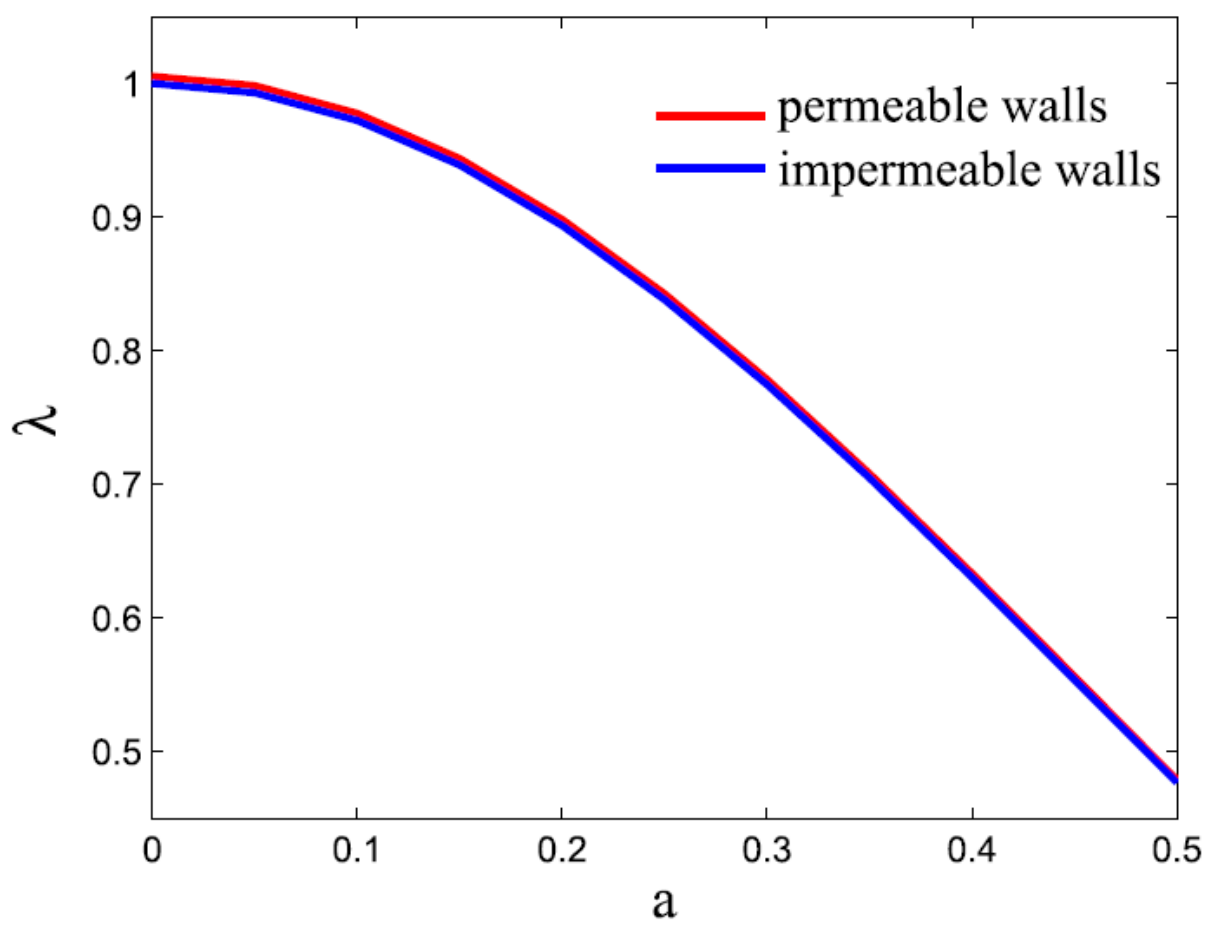

FIG. 21. Parallel-plate wavy channel: Dimensionless permeability $\lambda$ vs. wall oscillation amplitude $a$.

$$
\begin{gathered}
\beta^{\mathrm{ef}} \equiv 0, \\
\gamma^{\mathrm{ef}} \equiv \frac{12 \omega^{2}}{13475 \varepsilon^{2}} \frac{\left(39\left(\frac{\phi_{y y}^{\prime \prime}}{\phi^{2}}\right)-52\left(\frac{\phi_{y}^{\prime 2}}{\phi^{3}}\right)\right)}{\left\langle\frac{1}{\phi}\right)^{3}} .
\end{gathered}
$$

The dependence of these parameters, taken in dimensionless form, on the dimensionless amplitude of wall oscillations $a$ (the amplitude divided by the channel's aperture $H$ ), is shown in Figs. 21-23. It was assumed that $\omega=0.01, \varepsilon=0.01^{0.75}$. As can be seen in Fig. 21, the influence of the fluid flow through the walls on the 
permeability is not significant, and as is shown in Fig. 23, the viscous-inertias parameter is practically independent of the flow through the boundaries of channel. The pure inertia parameter $\beta^{\text {ef }}$ is the most sensible with respect to the fluid flow through the walls and increases when the walls are permeable, Fig. 22. Comparing Figs. 7(a) and 18(a) one can see that flow vorticities form at higher Reynolds in parallel-plate channels.

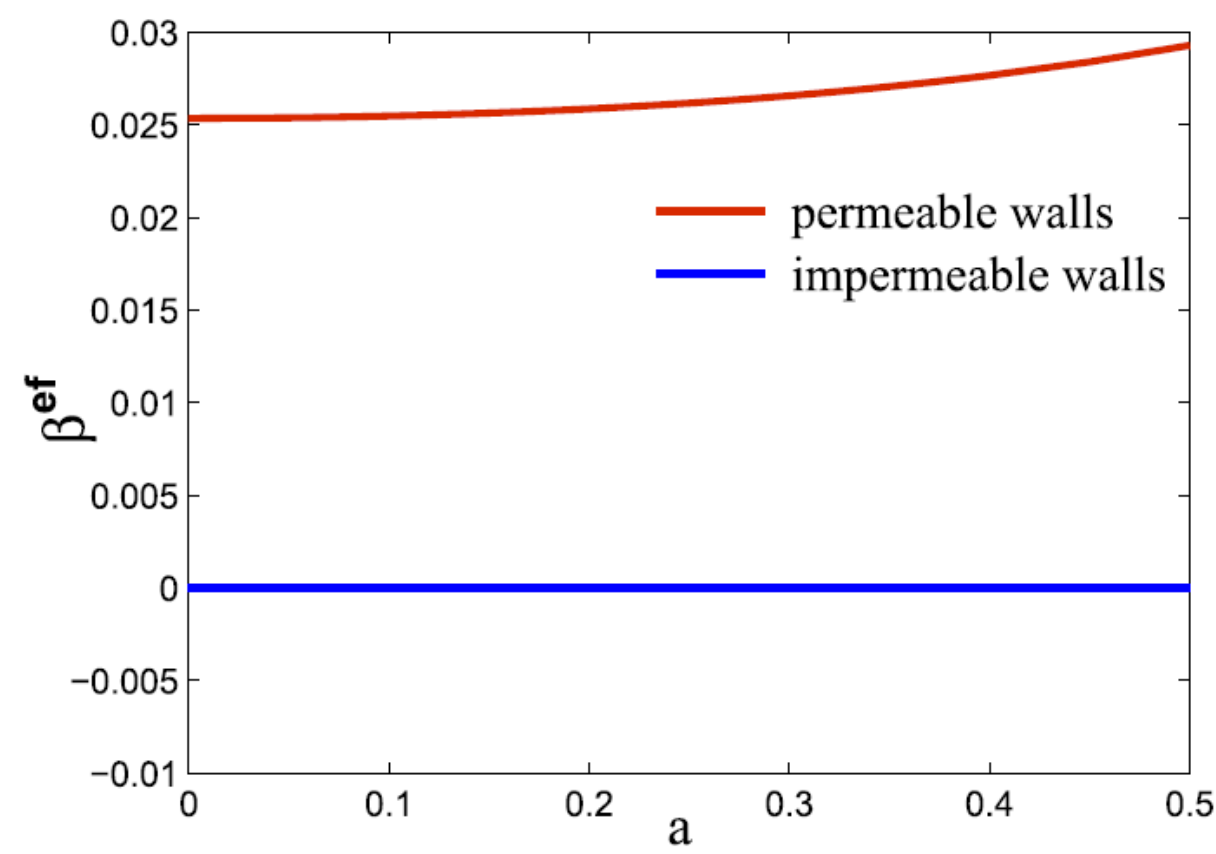

FIG. 22. Parallel-plate wavy channel: Dimensionless pure inertia coefficient $\beta^{\text {ef }} \cdot H$ vs. wall oscillation amplitude $a 1$.

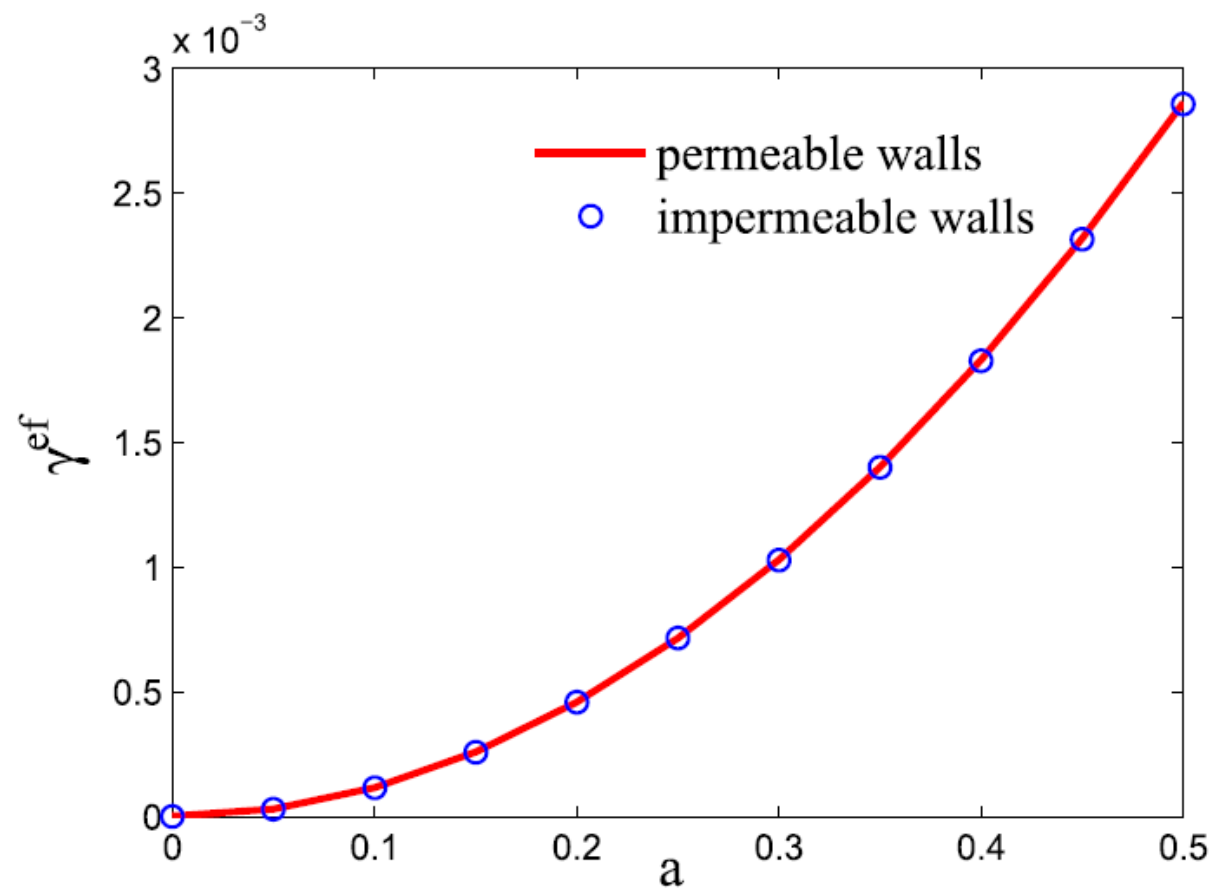

FIG. 23. Parallel-plate wavy channel: Dimensionless visco-inertial cross parameter $\gamma^{\text {ef }}$ vs. wall oscillation amplitude $a$.

Looking into Figs. 13 and 22, it is concluded that the dimensionless inertial coefficient $\beta^{\text {ef }}$ increases for parallelplate channels as the amplitude of the corrugations increase, while it decreases slightly for cylindrical-shape channels. The cross visco-inertial effects in a cylindrical channel decrease the pressure drop along the channel as the amplitude of corrugations increase, Fig. 14. For the slab-shaped channels, the cross visco-inertial coefficient is one order of magnitude smaller than the pure inertial effect and increases the pressure drop along the channel as the corrugation amplitude increases. 


\section{CONCLUSION}

The effective model of flow in a channel with permeable wavy walls was investigated through the asymptotic solutions of the steady-state Navier-Stokes equations with non-zero fluid exchange through the boundaries. The solution was obtained for a cylindrical and parallel-plate wavy channel. The fluid flow through the walls increases the Reynolds number along the channel and results in local flow instabilities and forms reverse flow. The local instabilities arise in relatively higher Reynolds numbers in parallel-plate wavy channels than in cylindrical wavy channels. The channel's effective permeability decreases as the amplitude of corrugations increases. It was shown that the linear approximation for proportionality of the average pressure gradient and average velocity cannot explain the effect of fluid flow through the walls and corrugated geometry of the channel. Consequently, the linear approximation cannot express the flow regime in the channel properly. The quadratic correction to pressure drop along the channel is valid only in case of permeable walls. When the channel walls are impermeable the quadratic term vanishes, and the cubic correction to linear pressure drop exists though due to the wavy geometry of the walls. In cylindrical wavy channels, the cubic term decreases the pressure drop along the channel as the corrugation amplitude increases, while in parallel-plate channels the cubic term is less dominant.

\section{APPENDIX A. ASYMPTOTIC SOLUTION IN CYLINDRICAL WAVY CHANNEL}

The zeroth order, first order, and second order problems given in Sec. II A may be solved by introducing new variables,

$$
(r, x) \rightarrow(\zeta, x), \zeta \equiv \frac{r}{\phi(x)} \text {. Then } \frac{\partial}{\partial r}=\frac{1}{\phi} \frac{\partial}{\partial \zeta}, \quad \frac{\partial}{\partial x}=-\frac{\zeta \phi^{\prime}}{\phi} \frac{\partial}{\partial \zeta} \text {, where } \phi^{\prime}=\frac{d \phi}{d x} \text {. }
$$

Then the zero-order problem of the asymptotic series becomes :

$$
\begin{gathered}
\frac{\partial p_{0}}{\partial \zeta}=0, \quad 0=-\frac{d p_{0}}{d x}+\frac{1}{\zeta \phi^{2}} \frac{\partial}{\partial \zeta}\left(\zeta \frac{\partial u_{0}}{\partial \zeta}\right), \\
\left.u_{0}\right|_{\zeta=1}=0,\left.\quad \frac{\partial u_{0}}{\partial \zeta}\right|_{\zeta=0}=0, \quad \phi^{2} \int_{0}^{1} \zeta u_{0}(x, \zeta) d \zeta=F_{0}(x) .
\end{gathered}
$$

In the second equation, the variables $x$ and $\zeta$ may be separated as

$$
\phi^{2}(x) \frac{d p_{0}}{d x}=\frac{1}{\zeta} \frac{\partial}{\partial \zeta}\left(\zeta \frac{\partial u_{0}}{\partial \zeta}\right)
$$

The left-hand side depends on $x$ only; then, this equation is easy to integrate while taking into account the boundary conditions $u_{0}=\frac{A(x)}{4}\left(\zeta^{2}-1\right), A(x) \equiv \phi^{2}(x) \frac{d p_{0}}{d x}$. From the last integral relation in Eq. (A1), we obtain

$$
\frac{A}{4} \phi^{2} \int_{0}^{1}\left(\zeta^{3}-\zeta\right) d \zeta=F_{0} .
$$

Then $A(x) \equiv \phi^{2} \frac{d p_{0}}{d x}=-16 \frac{F_{0}}{\phi^{2}}$. The solution takes the form 


$$
u_{0}=-4 \frac{F_{0}}{\phi^{2}}\left(\zeta^{2}-1\right), \quad \frac{d p_{0}}{d x}=-16 \frac{F_{0}}{\phi^{4}} .
$$

In a similar way, one can solve the first-order and second-order problem and obtain for

$$
\begin{aligned}
& u_{0}(x, \zeta)=-4 \frac{F_{0}}{\phi^{2}}\left(\zeta^{2}-1\right), \\
& u_{1}=u^{*}\left(1+\frac{2 \phi^{2}(0)}{\phi^{2}}\left(\zeta^{2}-1\right)\right)+\frac{\operatorname{Re} F_{0} v^{*}}{\phi}\left(\frac{2 \zeta^{6}}{9}-\zeta^{4}+\zeta^{2}-\frac{2}{9}\right)-\frac{\operatorname{Re} \phi^{\prime} F_{0}^{2}}{\phi^{3}}\left(\frac{8 \zeta^{6}}{9}-4 \zeta^{4}+4 \zeta^{2}-\frac{8}{9}\right) . \\
& u_{2}=\left(8\left(\frac{\phi^{\prime}}{\phi}\right)^{2} F_{0}-2 \frac{\phi^{\prime \prime} F_{0}}{\phi}+\frac{\operatorname{Re} \phi^{\prime} F_{0} u^{*}}{\phi}-3 v^{*} \phi^{\prime}-\operatorname{Re} \frac{u^{*} v^{*} \phi}{4}\right)\left(\zeta^{4}-\frac{4}{3} \zeta^{2}+\frac{1}{3}\right) \\
& +\left(\frac{8 \operatorname{Re} \phi^{\prime} F_{0} u^{*}}{\phi^{3}}-\frac{\operatorname{Re} u^{*} v^{*}}{\phi}\right)\left(\frac{\zeta^{6}}{9}-\frac{\zeta^{4}}{2}+\frac{\zeta^{2}}{2}-\frac{1}{9}\right)-\operatorname{Re}^{2} v^{* 2} F_{0}\left(\frac{\zeta^{10}}{450}-\frac{\zeta^{8}}{36}+\frac{\zeta^{6}}{9}-\frac{11}{36} \zeta^{-4}+\frac{38}{135} \zeta^{2}-\frac{83}{1350}\right) \\
& +\frac{\operatorname{Re}^{2} \phi^{\prime} F_{0}^{2} v^{*}}{\phi^{2}}\left(\frac{29}{450} \zeta^{10}-\frac{7}{12} \zeta^{8}+\frac{11}{6} \zeta^{6}-\frac{115}{36} \zeta^{4}+\frac{1261}{540} \zeta^{2}-\frac{1229}{2700}\right) \\
& +\frac{\operatorname{Re}^{2} \phi^{\prime \prime} F_{0}^{3}}{\phi^{3}}\left(\frac{2}{75} \zeta^{10}-\frac{2}{9} \zeta^{8}+\frac{2}{3} \zeta^{6}-\zeta^{4}+\frac{29}{45} \zeta^{2}-\frac{26}{225}\right)-\frac{\operatorname{Re}^{2} \phi^{\prime 2} F_{0}^{3}}{\phi^{4}}\left(\frac{38}{225} \zeta^{10}-\frac{13}{9} \zeta^{8}+\frac{38}{9} \zeta^{6}-\frac{53}{9} \zeta^{4}+\frac{479}{135} \zeta^{2}-\frac{409}{675}\right) . \\
& v_{1}=v^{*}\left(\zeta^{3}-2 \zeta\right)-\frac{4 \phi^{\prime} F_{0}}{\phi^{2}}\left(\zeta^{3}-\zeta\right) . \\
& v_{2}=\frac{2 u^{*} \phi^{\prime} \phi^{2}(0)}{\phi^{2}}\left(\zeta^{3}-\zeta\right) \\
& +\left(\frac{\operatorname{Re} \phi^{\prime \prime} F_{0}^{2}}{\phi^{2}}-\frac{\operatorname{Rev} v^{* 2} \phi}{4}\right)\left(\frac{\zeta^{7}}{9}-\frac{2 \zeta^{5}}{3}+\zeta^{3}-\frac{4 \zeta}{9}\right) \\
& -\frac{\operatorname{Re} \phi^{2} F_{0}^{2}}{\phi^{3}}\left(\zeta^{7}-\frac{14 \zeta^{5}}{3}+5 \zeta^{3}-\frac{4 \zeta}{3}\right) \\
& +\frac{\operatorname{Re} F_{0} \phi^{\prime} v^{*}}{\phi}\left(\frac{5 \zeta^{7}}{12}-\frac{13 \zeta^{5}}{6}+\frac{11 \zeta^{3}}{4}-\zeta\right) . \quad(\mathrm{A} 4 \mathrm{e} \\
& \frac{d p_{0}}{d x}=-16 \frac{F_{0}}{\phi^{4}} \text {. } \\
& \frac{\partial p_{1}}{\partial x}=\frac{8 u^{*} \phi^{2}(0)}{\phi^{4}}-\frac{12 \operatorname{Re} F_{0} v^{*}}{\phi^{3}}+\frac{16 \operatorname{Re} \phi^{\prime} F_{0}^{2}}{\phi^{5}}, \\
& \frac{\partial p_{2}}{\partial x}=16\left(-\frac{5 \phi^{\prime} v^{*}}{4 \phi^{2}}-\frac{\phi^{\prime \prime} F_{0}}{\phi^{3}}+3 \frac{\phi^{2} F_{0}}{\phi^{4}}\right) \zeta^{2}+\frac{8}{3} \frac{\phi^{\prime \prime} F_{0}}{\phi^{3}} \\
& -\frac{56}{3} \frac{\phi^{\prime 2} F_{0}}{\phi^{4}}+\frac{4 v^{*} \phi^{\prime}}{\phi^{2}}+\frac{8 \operatorname{Re} u^{*} \phi^{\prime} F_{0}}{3 \phi^{3}}+\frac{6 \operatorname{Re} u^{*} v^{*}}{\phi^{3}} \\
& -\frac{16 \operatorname{Re} u^{*} \phi^{\prime} F_{0}}{\phi^{5}}-\frac{8 \operatorname{Re} u^{*} v^{*}}{3 \phi}-\frac{44 \operatorname{Re}^{2} \phi^{\prime \prime}}{45 \phi^{5}}\left(F_{0}^{3}-1\right) \\
& +\frac{484 \operatorname{Re}^{2} \phi^{2} F_{0}^{3}}{135 \phi^{6}}+\frac{88 \operatorname{Re}^{2} v^{-2} F_{0}}{135 \phi^{2}}-\frac{539 \operatorname{Re}^{2} \phi^{\prime} v^{*}}{135 \phi^{4}}\left(F_{0}^{2}-1\right) .
\end{aligned}
$$




\section{APPENDIX B: TWO-SCALE FORM OF THE SOLUTION IN CYLINDRICAL WAVY CHANNEL}

The two-scale form of Eqs. (A4) will be written as

$$
\begin{aligned}
& u=-\frac{4 F_{0}}{\phi^{2}}\left(\zeta^{2}-1\right)+\frac{\omega}{\varepsilon}\left\{-\frac{8 \operatorname{Re} \phi_{y}^{\prime} F_{0}^{2}}{\phi^{3}}\left(\frac{\zeta^{6}}{9}-\frac{\zeta^{4}}{2}+\frac{\zeta^{2}}{2}-\frac{1}{9}\right)+\varepsilon\left[\frac{2 \operatorname{Re} v^{*} F_{0}}{\phi}\left(\frac{\zeta^{6}}{9}-\frac{\zeta^{4}}{2}+\frac{\zeta^{2}}{2}-\frac{1}{9}\right)\right.\right. \\
& \left.\left.+u^{*}\left(1+\frac{2 \phi^{2}(0)}{\phi^{2}}\left(\zeta^{2}-1\right)\right)\right]+\varepsilon^{2} \cdots\right\}+\frac{\omega^{2}}{\varepsilon^{2}}\left\{\left(8\left(\frac{\phi_{y}^{\prime}}{\phi}\right)^{2} F_{0}-2 \frac{\phi_{y y}^{\prime \prime} F_{0}}{\phi}\right)\left(\zeta^{4}-\frac{4}{3} \zeta^{2}+\frac{1}{3}\right)\right. \\
& +\frac{\operatorname{Re}^{2} \phi_{y y}^{\prime \prime} F_{0}^{3}}{\phi^{3}}\left(\frac{2}{75} \zeta^{10}-\frac{2}{9} \zeta^{8}+\frac{2}{3} \zeta^{6}-\zeta^{4}+\frac{29}{45} \zeta^{2}-\frac{26}{225}\right)-\frac{\operatorname{Re}^{2} \phi_{y}^{\prime 2} F_{0}^{3}}{\phi^{4}}\left(\frac{38}{225} \zeta^{10}-\frac{13}{9} \zeta^{8}+\frac{38}{9} \zeta^{6}-\frac{53}{9} \zeta^{4}+\frac{479}{135} \zeta^{2}-\frac{409}{675}\right) \\
& +\varepsilon\left[\left(\frac{\operatorname{Re} \phi_{y}^{\prime} F_{0} u^{*}}{\phi}-3 v^{*} \phi_{y}^{\prime}\right)\left(\zeta^{+}-\frac{4}{3} \zeta^{2}+\frac{1}{3}\right)+\frac{8 \operatorname{Re} \phi_{y}^{\prime} F_{0} u^{*}}{\phi^{3}}\left(\frac{\zeta^{6}}{9}-\frac{\zeta^{4}}{2}+\frac{\zeta^{2}}{2}-\frac{1}{9}\right)\right. \\
& \left.+\frac{\operatorname{Re}^{2} \phi_{y}^{\prime} F_{0}^{2} v^{*}}{\phi^{2}}\left(\frac{29}{450} \zeta^{10}-\frac{7}{12} \zeta^{8}+\frac{11}{6} \zeta^{6}-\frac{115}{36} \zeta^{4}+\frac{1261}{540} \zeta^{2}-\frac{1229}{2700}\right)\right] \\
& +\varepsilon^{2}\left[-\operatorname{Re} \frac{u^{*} v^{*} \phi}{4}\left(\zeta^{4}-\frac{4}{3} \zeta^{2}+\frac{1}{3}\right)-\frac{\operatorname{Re} u^{*} v^{*}}{\phi}\left(\frac{\zeta^{6}}{9}-\frac{\zeta^{4}}{2}+\frac{\zeta^{2}}{2}-\frac{1}{9}\right)\right. \\
& \left.\left.-\operatorname{Re}^{2} v^{* 2} F_{0}\left(\frac{\zeta^{10}}{450}-\frac{\zeta^{8}}{36}+\frac{\zeta^{6}}{9}-\frac{11}{36} \zeta^{4}+\frac{38}{135} \zeta^{2}-\frac{83}{1350}\right)\right]+\varepsilon^{3} \cdots\right\}+O\left(\frac{\omega^{3}}{\varepsilon^{3}}+\omega \varepsilon\right), \\
& v=\frac{\omega}{\varepsilon}\left\{-\frac{4 \phi_{y}^{\prime} F_{0}}{\phi^{2}}\left(\zeta^{3}-\zeta\right)+\varepsilon v^{*}\left(\zeta^{3}-2 \zeta\right)+\varepsilon^{2} \cdots\right\}+\frac{\omega^{2}}{\varepsilon^{2}}\left\{\frac{\operatorname{Re} \phi_{y}^{\prime \prime} F_{0}^{2}}{\phi^{2}}\left(\frac{\zeta^{7}}{9}-\frac{2 \zeta^{5}}{3}+\zeta^{3}-\frac{4 \zeta}{9}\right)\right. \\
& -\frac{\operatorname{Re} \phi_{y}^{\prime 2} F_{0}^{2}}{\phi^{3}}\left(\zeta^{7}-\frac{14 \zeta^{5}}{3}+5 \zeta^{3}-\frac{4 \zeta}{3}\right)+\varepsilon\left[\frac{2 u^{*} \phi_{y}^{\prime} \phi^{2}(0)}{\phi^{2}}\left(\zeta^{3}-\zeta\right)+\frac{\operatorname{Re} F_{0} \phi_{y}^{\prime} v^{*}}{\phi}\left(\frac{5 \zeta^{7}}{12}-\frac{13 \zeta^{5}}{6}+\frac{11 \zeta^{3}}{4}-\zeta\right)\right] \\
& \left.+\varepsilon^{2}\left[-\frac{\operatorname{Re} v^{* 2} \phi}{4}\left(\frac{\zeta^{7}}{9}-\frac{2 \zeta^{5}}{3}+\zeta^{3}-\frac{4 \zeta}{9}\right)\right]+\varepsilon^{3} \cdots+O\left(\frac{\omega^{3}}{\varepsilon^{3}}+\omega \varepsilon\right)\right\} \text {. } \\
& \frac{\partial p}{\partial x}=-16 \frac{F_{0}}{\phi^{4}}+\frac{\omega}{\varepsilon}\left\{\frac{16 \operatorname{Re} \phi^{\prime} F_{0}^{2}}{\phi^{5}}+\varepsilon\left[8 u^{*} \frac{\phi^{2}(0)}{\phi^{4}}-\frac{12 \operatorname{Re} F_{0} v^{*}}{\phi^{3}}\right]+\varepsilon^{2} \cdots\right\} \\
& +\frac{\omega^{2}}{\varepsilon^{2}}\left\{16\left(-\frac{\phi^{\prime \prime} F_{0}}{\phi^{3}}+3 \frac{\phi^{\prime 2} F_{0}}{\phi^{4}}\right) \zeta^{2}+\frac{8}{3} \frac{\phi^{\prime \prime} F_{0}}{\phi^{3}}-\operatorname{Re}^{2} \frac{44}{45} \frac{\phi^{\prime \prime}}{\phi^{5}}\left(F_{0}^{3}-1\right)-\frac{56}{3} \frac{\phi^{\prime 2} F_{0}}{\phi^{4}}+\operatorname{Re}^{2} \frac{484}{135} \frac{\phi^{\prime 2} F_{0}^{3}}{\phi^{6}}\right. \\
& +\varepsilon\left[-20 \frac{\phi^{\prime} v^{*}}{\phi^{2}} \zeta^{2}+4 \frac{v^{*} \phi^{\prime}}{\phi^{2}}-\operatorname{Re}^{2} \frac{539}{135} \frac{\phi^{\prime} v^{*}}{\phi^{4}}\left(F_{0}^{2}-1\right)+\operatorname{Re} \frac{8}{3} \frac{u^{*} \phi^{\prime} F_{0}}{\phi^{3}}-16 \operatorname{Re} \frac{u^{*} \phi^{\prime} F_{0}}{\phi^{5}}\right] \\
& \left.+\varepsilon^{2}\left[-\operatorname{Re} \frac{8}{3} \frac{u^{*} v^{*}}{\phi}+6 \operatorname{Re} \frac{u^{*} v^{*}}{\phi^{3}}+\operatorname{Re}^{2} \frac{88}{135} \frac{v^{* 2} F_{0}}{\phi^{2}}\right]+\varepsilon^{3} \cdots\right\}+O\left(\frac{\omega^{3}}{\varepsilon^{3}}+\omega \varepsilon\right) .
\end{aligned}
$$

In the cylindrical coordinating system, considering the symmetry with respect to the angular coordinate, the streamlines in terms of radial and longitudinal velocity components, respectively, $u, v$ can be written as

$$
u=-\frac{1}{r} \frac{\partial \psi}{\partial r}, \quad v=\frac{1}{r} \frac{\partial \psi}{\partial x} .
$$


This is

$$
\begin{aligned}
\psi= & F_{0}\left(\zeta^{4}-2 \zeta^{2}\right)+\frac{\omega}{\varepsilon} \frac{8 \operatorname{Re} \phi^{\prime} F_{0}^{2}}{\phi}\left(\frac{\zeta^{8}}{9}-\frac{2 \zeta^{6}}{3}+\zeta^{4}-\frac{4 \zeta^{2}}{9}\right)+\frac{\omega^{2}}{\delta^{2}}\left\{\left(-4 \phi^{\prime 2} F_{0}+\phi^{\prime \prime} \phi F_{0}\right)\left(\frac{\zeta^{6}}{3}-\frac{2}{3} \zeta^{4}+\frac{\zeta^{2}}{3}\right)\right. \\
& -\frac{\operatorname{Re}^{2} \phi^{\prime \prime} F_{0}^{3}}{\phi}\left(\frac{\zeta^{12}}{450}-\frac{\zeta^{10}}{45}+\frac{\zeta^{8}}{12}-\frac{\zeta^{6}}{6}+\frac{29}{180} \zeta^{4}-\frac{13}{225} \zeta^{2}\right) \\
& \left.+\frac{\operatorname{Re}^{2} \phi^{\prime 2} F_{0}^{3}}{\phi^{2}}\left(\frac{38}{2700} \zeta^{12}-\frac{13 \zeta^{10}}{90}+\frac{38 \zeta^{8}}{72}-\frac{53 \zeta^{6}}{54}+\frac{479 \zeta^{4}}{540}-\frac{409 \zeta^{2}}{1350}\right)\right\}+C+O\left(\omega^{3}\right) .
\end{aligned}
$$

where $C$ is an arbitrary constant, representing different streamline sets. It should be noted that the function $\phi$ oscillates with respect to $y$ and does not depend on $x$, so one can write $d / d x=(1 / \varepsilon) d / d y$.

\section{APPENDIX C: TWO-SCALE FORM OF THE SOLUTION IN PARALLEL-PLATE WAVY CHANNEL}

The two-scale asymptotic solutions for flow in a parallel-plate wavy channel will be derived in the similar way as cylindrical wavy channel given in Appendixes A and B, which rewrite to

$$
\begin{aligned}
& u=-\frac{3}{2} \frac{F_{0}}{\phi}\left(\zeta^{2}-1\right)+\frac{\omega}{\varepsilon}\left\{-\frac{3 \operatorname{Re} F_{0}^{2} \phi_{y}^{\prime}}{280 \phi}\left(7 \zeta^{6}-35 \zeta^{4}+33 \zeta^{2}-5\right)+\varepsilon\left[\frac{\operatorname{Re} F_{0} v^{*}}{280}\left(7 \zeta^{6}-9 \zeta^{2}+2\right)+\frac{3 u^{*} \phi(0)}{2 \phi}\left(\zeta^{2}-1\right)+u^{*}\right]+\varepsilon^{2} \cdots\right\} \\
& +\frac{\omega^{2}}{\varepsilon^{2}}\left\{\frac{1}{20}\left(-3 F_{0} \phi_{y y}^{\prime \prime}+\frac{9 F_{0} \phi_{y}^{2}}{\phi}\right)\left(5 \zeta^{4}-6 \zeta^{2}+1\right)+\frac{\operatorname{Re}^{2} F_{0}^{3} \phi_{y y}^{\prime \prime}}{1120}\left(\zeta^{10}-9 \zeta^{8}+\frac{138}{5} \zeta^{-6}-42 \zeta^{4}+\frac{9837}{385} \zeta^{2}-\frac{1213}{385}\right)\right. \\
& -\frac{\operatorname{Re}^{2} F_{0}^{3} \phi_{y}^{\prime 2}}{280 \phi}\left(\frac{7}{10} \zeta^{10}-\frac{27}{4} \zeta^{8}+\frac{102}{5} \zeta^{6}-\frac{57}{2} \zeta^{4}+\frac{12333}{770} \zeta^{2}-\frac{575}{313}\right) \\
& +s\left[-\frac{1}{4}\left(v^{*} \phi_{y}^{\prime}\right)\left(5 \zeta^{4}-6 \zeta^{2}+1\right)+\frac{\operatorname{Re} u^{*} \phi_{y}^{\prime} F_{0}}{280}\left(105 \zeta^{-4}-126 \zeta^{2}+21\right)+\frac{3 \operatorname{Re} u^{*} \phi(0) \phi_{y}^{\prime} F_{0}}{140 \phi}\left(7 \zeta^{6}-35 \zeta^{-4}+33 \zeta^{2}-5\right)\right. \\
& \left.+\frac{\operatorname{Re}^{2} v^{*} F_{0}^{2} \phi_{y}^{\prime}}{5600}\left(6 \zeta^{10}-\frac{75}{4} \zeta^{8}-21 \zeta^{6}-1725 \zeta^{4}+\frac{2759}{11} \zeta^{2}-\frac{1961}{44}\right)\right]+\varepsilon^{2}\left[-\frac{\operatorname{Re} u^{*} v^{*} \phi(0)}{280}\left(7 \zeta^{6}-9 \zeta^{2}+2\right)\right. \\
& \left.\left.-\frac{\operatorname{Re} u^{*} v^{*} \phi}{40}\left(5 \zeta^{4}-6 \zeta^{2}+1\right)+\frac{\operatorname{Re}^{2} v^{* 2} F_{0} \phi}{2800}\left(\frac{\zeta^{10}}{3}-\frac{15}{2} \zeta^{8}+3 \zeta^{6}+\frac{438}{77} \zeta^{2}-\frac{703}{462}\right)\right]+\varepsilon^{3} \cdots\right\}+O\left(\frac{\omega^{3}}{\varepsilon^{3}}+\omega \varepsilon\right) . \\
& v=\frac{\omega}{\varepsilon}\left\{-\frac{3 F_{0} \phi_{y}^{\prime}}{2 \phi}\left(\zeta^{3}-\zeta\right)+\varepsilon \frac{3 v^{*}}{2}\left(\frac{\zeta^{3}}{3}-\zeta\right)+\varepsilon^{2} \cdots\right\}+\frac{\omega^{2}}{\varepsilon^{2}}\left\{-\frac{3 \operatorname{Re} \phi_{y}^{\prime 2} F_{0}^{2}}{280 \phi}\left(7 \zeta^{7}-35 \zeta^{5}+33 \zeta^{3}-5 \zeta\right)\right. \\
& +\frac{3 \operatorname{Re} \phi_{y y}^{\prime \prime} F_{0}^{2}}{280}\left(\zeta^{7}-7 \zeta^{5}+11 \zeta^{3}-5 \zeta\right)+\varepsilon\left[\frac{3 u^{*} \phi_{y}^{\prime} \phi(0)}{2 \phi}\left(\zeta^{3}-\zeta\right)+\frac{3 \operatorname{Re} v^{*} \phi_{y}^{\prime} F_{0}}{140}\left(2 \zeta^{7}-7 \zeta^{5}+10 \zeta^{3}-5 \zeta\right)\right] \\
& \left.+\varepsilon^{2}\left[\frac{\operatorname{Re} \phi v^{* 2}}{280}\left(-\zeta^{7}+3 \zeta^{3}-2 \zeta\right)\right]+\varepsilon^{3} \cdots\right\}+O\left(\frac{\omega^{3}}{\varepsilon^{3}}+\omega \varepsilon\right) \text {. } \\
& \frac{\partial p}{\partial x}=-\frac{3 F_{0}}{\phi^{3}}+\frac{\omega}{\varepsilon}\left\{\frac{54 \operatorname{Re} \phi_{y}^{\prime} F_{0}^{2}}{35 \phi^{3}}+\varepsilon\left[\frac{3 u^{*} \phi(0)}{\phi^{3}}-\frac{81 \operatorname{Re} v^{*} F_{0}}{35 \phi^{2}}\right]+\varepsilon^{2} \cdots\right\}+\frac{\omega^{2}}{\varepsilon^{2}}\left\{\left(\frac{9 \phi_{y}^{\prime 2} F_{0}}{\phi^{3}}-\frac{9 \phi_{y y}^{\prime \prime} F_{0}}{2 \phi^{2}}\right) \zeta^{2}+\frac{3 \phi_{y y}^{\prime \prime} F_{0}}{10 \phi^{2}}-\frac{12 \phi_{y}^{\prime 2} F_{0}}{5 \phi^{3}}\right. \\
& +\frac{156 \operatorname{Re}^{2} F_{0}^{3}}{13475}\left(\frac{4 \phi_{y}^{2}}{\phi^{3}}-\frac{3 \phi_{y y}^{\prime \prime}}{\phi^{2}}\right)+\varepsilon\left[-\frac{6 v^{*} \phi^{\prime}}{\phi^{2}} \zeta^{2}+\frac{3 \operatorname{Re} u^{*} \phi_{y}^{\prime} F_{0}}{5 \phi^{2}}-\frac{271 \operatorname{Re}^{2} v^{*} \phi_{y}^{\prime} F_{0}^{2}}{1925 \phi^{2}}-\frac{108 \operatorname{Re} u^{*} \phi(0) \phi_{y}^{\prime} F_{0}}{35 \phi^{3}}\right] \\
& \left.+s^{2}\left[\frac{81 \operatorname{Re} u^{*} v^{*} \phi(0)}{35 \phi^{2}}-\frac{6 \operatorname{Re} u^{*} v^{*}}{5 \phi}-\frac{234 \operatorname{Re}^{2} v^{* 2} F_{0}}{13475 \phi}\right]+\varepsilon^{3} \cdots\right\}+O\left(\frac{\omega^{3}}{\varepsilon^{3}}+\omega \varepsilon\right) \text {. }
\end{aligned}
$$




$$
\begin{aligned}
\psi= & -\frac{3}{2} F_{0}\left(\frac{\zeta^{3}}{3}-\zeta\right)+\omega\left\{-\frac{3}{280} \operatorname{Re} F_{0}^{2} \phi^{\prime}\left(\zeta^{7}-7 \zeta^{5}+11 \zeta^{3}-5 \zeta\right)\right\}+\omega^{2}\left\{\left(-3 F_{0} \phi \phi^{\prime \prime}+9 F_{0} \phi^{\prime 2}\right)\left(\frac{\zeta^{5}}{20}-\frac{\zeta^{3}}{10}+\frac{\zeta}{20}\right)\right. \\
& +\frac{1}{280} \operatorname{Re}^{2} F_{0}^{3} \phi \phi^{\prime \prime}\left(\frac{\zeta^{11}}{44}-\frac{\zeta^{9}}{4}+\frac{69}{70} \zeta^{7}-\frac{21}{10} \zeta^{5}+\frac{3279}{154} \zeta^{3}-\frac{1213}{154} \zeta\right) \\
& \left.-\frac{1}{280} \operatorname{Re}^{2} F_{0}^{3} \phi^{\prime 2}\left(\frac{7 \zeta^{11}}{110}-\frac{3}{4} \zeta^{9}+\frac{204}{70} \zeta^{7}-\frac{57}{10} \zeta^{5}+\frac{4111}{770} \zeta^{3}-\frac{575}{313} \zeta\right)\right\}+C+O\left(\omega^{3}\right) .
\end{aligned}
$$

where $C$ is an arbitrary constant.

\section{REFERENCES}

Adler, P. M. and Thovert, J.-F., Fractures and Fracture Networks (Springer Science \& Business Media, 1999), Vol. 15.

Adler, P., Malevich, A., and Mityushev, V., "Nonlinear correction to Darcy’s law for channels with wavy walls," Acta Mech. 224, 1823 (2013).

Bennett, C. O. and Myers, J. E., Momentum, Heat, and Mass Transfer (McGraw-Hill, New York, 1982), Vol. 370.

Berman, A. S., "Laminar flow in channels with porous walls," J. Appl. Phys. 24, 1232 (1953).

Berman, A. S., "Effects of porous boundaries on the flow of fluids in systems with various geometries," Technical Report No. A/CONF. 15/P/720, Oak Ridge Gaseous Diffusion Plant, Tennessee, 1958.

Brush, D. J. and Thomson, N. R., "Fluid flow in synthetic rough-walled fractures: Navier-Stokes, Stokes, and local cubic law simulations," Water Resour. Res. 39, 1085, https://doi.org/10.1029/2002wr001346 (2003)

Bues, M., Panilov, M., Crosnier, S., and Oltean, C., "Macroscale model and viscous-inertia effects for Navier-Stokes flow in a radial fracture with corrugated walls," J. Fluid Mech. 504, 41 (2004).

Burns, J. and Parkes, T., "Peristaltic motion,” J. Fluid Mech. 29, 731 (1967). Chen, C.-P., "Contribution a letude experimentale de lecoulement radial dun fluide visqueux incompressible entre deux disques paralleles," J. Mec. 5, 245 (1966).

Chen, Z., Lyons, S. L., and Qin, G., “Derivation of the Forchheimer law via homogenization,” Transp. Porous Media 44, 325 (2001).

Crosnier, S., Chevalier, S., and Bu`es, M., "Comparison between numerical simulations and experimental investigations of radial flows in rough fracture," WIT Trans. Eng. Sci. 29, 43-52 (1970).

Deiber, J. and Schowalter, W., "Flow through tubes with sinusoidal axial variations in diameter," AIChE J. 25, 638 (1979).

Forchheimer, P., "Wasserbewegung durch Boden,” WDIZ 45, 1782 (1901).

Ge, S., “A governing equation for fluid flow in rough fractures," Water Resour. Res. 33, 53, https://doi.org/10.1029/96wr02588 (1997).

Haroun, M. H., "Non-linear peristaltic flow of a fourth grade fluid in an inclined asymmetric channel," Comput. Mater. Sci. 39, 324 (2007).

Heining, C., Pollak, T., and Aksel, N., "Pattern formation and mixing in threedimensional film flow," Phys. Fluids 24, 042102 (2012).

Ihara, M., Kikuyama, K., Mizuguchi, K. et al., in SPE Annual Technical Conference and Exhibition (Society of Petroleum Engineers, 1994).

Katsuhiko, I., Fundamental Studies of Fluid Flow through a Single Fracture (University Microfilms, 1976).

Keller, A., "High resolution, non-destructive measurement and characterization of fracture apertures," Int. J. Rock Mech. Min. Sci. 35, 1037 (1998).

Konzuk, J. S. and Kueper, B. H., "Evaluation of cubic law based models describing single-phase flow through a rough-walled fracture," Water Resour. Res. 40, W02402, https://doi.org/10.1029/2003wr002356 (2004).

Lahbabi, A. and Chang, H.-C., "Flow in periodically constricted tubes: Transition to inertial and nonsteady flows," Chem. Eng. Sci. 41, 2487 (1986).

Landau, L. D., Hydrodynamics (Akademie-Verlag, 1974).

Malevich, A., Mityushev, V., and Adler, P., "Stokes flow through a channel with wavy walls," Acta Mech. 182, 151 (2006).

Manton, M., "Low Reynolds number flow in slowly varying axisymmetric tubes," J. Fluid Mech. 49, 451 (1971).

Mei, C. and Auriault, J.-L., "The effect of weak inertia on flow through a porous medium," J. Fluid Mech. 222, 647 (1991).

Mourzenko, V., Thovert, J.-F., and Adler, P., Transport in Porous Media (Springer, 2018), p. 1.

Nishimura, T., Ohori, Y., and Kawamura, Y., "Flow characteristics in a channel with symmetric wavy wall for steady flow," J. Chem. Eng. Jpn. 17, 466 (1984).

Oron, A. P. and Berkowitz, B., "Flow in rock fractures: The local cubic law assumption reexamined," Water Resour. Res. 34, 2811, https://doi.org/10.1029/98wr02285 (1998).

Ouyang, L.-B., "Single phase and multiphase fluid flow in horizontal wells," Ph.D. dissertation (Stanford University, 1998).

Ouyang, L.-B., Arbabi, S., Aziz, K. et al., “General wellbore flow model for horizontal, vertical, and slanted well completions,” SPE J. 3, 124 (1998).

Panfilov, M. and Fourar, M., "Physical splitting of nonlinear effects in highvelocity stable flow through porous media," Adv. Water Resour. 29, 30 (2006).

Pfitzner, J., “Poiseuille and his law,” Anaesthesia 31, 273 (1976). 
Proudman, I., “An example of steady laminar flow at large Reynolds number,” J. Fluid Mech. 9, 593 (1960).

Renshaw, C. E., "On the relationship between mechanical and hydraulic apertures in rough-walled fractures,” J. Geophys. Res.: Solid Earth 100, 24629, https://doi.org/10.1029/95jb02159 (1995).

Robinson, W., "The existence of multiple solutions for the laminar flow in a uniformly porous channel with suction at both walls," J. Eng. Math. 10, 23 (1976).

Savage, S., "Laminar radial flow between parallel plates,” J. Appl. Mech. 31, 594 (1964).

Sellars, J. R., "Laminar flow in channels with porous walls at high suction Reynolds numbers," J. Appl. Phys. 26, 489 (1955).

Shenoy, A., Sheremet, M., and Pop, I., Convective Flow and Heat Transfer fromWavy Surfaces: Viscous Fluids, Porous Media, and Nanofluids (CRC Press, 2016).

Sisavath, S., Jing, X., and Zimmerman, R.W., "Creeping flow through a pipe of varying radius,” Phys. Fluids 13, 2762 (2001).

Sisavath, S., Al-Yaarubi, A., Pain, C. C., and Zimmerman, R. W., Thermo-Hydro-Mechanical Coupling in Fractured Rock (Springer, 2003), pp. 10091022

Skjetne, E. and Auriault, J., "New insights on steady, non-linear flow in porous media,” Eur. J. Mech. B: Fluids 18, 131 (1999).

Skjetne, E., Hansen, A., and Gudmundsson, J., "High-velocity flow in a rough fracture,” J. Fluid Mech. 383, 1 (1999).

Sureshkumar, R., "Local linear stability characteristics of viscoelastic periodic channel flow,” J. Non-Newtonian Fluid Mech. 97, 125 (2001).

Sutera, S. P. and Skalak, R., “The history of Poiseuille's law,” Annu. Rev. Fluid Mech. 25, 1 (1993).

Wah, T., "Laminar flow in a uniformly porous channel," Aeronaut. Q. 15, 299 (1964).

Whitaker, S., "The Forchheimer equation: A theoretical development,” Transp. Porous Media 25, 27 (1996).

White, Jr., F., Barfield, B., and Goglia, M., “Laminar flow in a uniformly porous channel,” J. Appl. Mech. 25, 613 (1958).

Wierschem, A., Scholle, M., and Aksel, N., "Vortices in film flowover strongly undulated bottom profiles at low Reynolds numbers," Phys. Fluids 15, 426 (2003).

Yuan, S., "Laminar pipe flow with injection and suction through a porous wall," Technical Report No. TR-PIB-25-P, James Forrestal Research Center, Princeton University, 1955.

Yuan, S., "Further investigation of laminar flow in channels with porous walls," J. Appl. Phys. 27, 267 (1956).

Zaturska, M., Drazin, P., and Banks, W., “On the flow of a viscous fluid driven along a channel by suction at porous walls,” Fluid Dyn. Res. 4, 151 (1988).

Zimmerman, R. and Bodvarsson, G., "Effective transmissivity of twodimensional fracture networks,” Int. J. Rock Mech. Mining Sciences Geomech. Abstr. 33(4), 433-438 (1996a).

Zimmerman, R. W. and Bodvarsson, G. S., "Hydraulic conductivity of rock fractures,” Transp. Porous Media 23, 1 (1996b).

Zimmerman, R. W. and Yeo, I.-W., Dynamics of Fluids in Fractured Rock (Wiley Online Library, 2000), p. 213. 